\title{
Jet pair production in POWHEG
}

\author{
Simone Alioli, ${ }^{a}$ Keith Hamilton, ${ }^{b}$ Paolo Nason, ${ }^{b}$ Carlo Oleari ${ }^{c}$ and Emanuele $\mathbf{R e}^{d}$ \\ ${ }^{a}$ Deutsches Elektronen-Synchrotron DESY, \\ Platanenallee 6, D-15738 Zeuthen, Germany \\ ${ }^{b}$ INFN, Sezione di Milano-Bicocca, \\ Piazza della Scienza 3, 20126 Milan, Italy \\ ${ }^{c}$ Università di Milano-Bicocca and INFN, Sezione di Milano-Bicocca, \\ Piazza della Scienza 3, 20126 Milan, Italy \\ ${ }^{d}$ Institute for Particle Physics Phenomenology, Department of Physics \\ University of Durham, Durham, DH1 3LE, U.K. \\ E-mail: simone.alioli@desy.de, Keith.Hamilton@mib.infn.it, \\ Paolo.Nason@mib.infn.it, Carlo.0leari@mib.infn.it, \\ emanuele.re@durham.ac.uk
}

ABSTRACT: We present an implementation of the next-to-leading order dijet production process in hadronic collisions in the framework of POWHEG, which is a method to implement NLO calculations within a shower Monte Carlo context. In constructing the simulation, we have made use of the POWHEG BOX toolkit, which makes light of many of the most technical steps. The majority of this article is concerned with the study of the predictions of the Monte Carlo simulation. In so doing, we validate our program for use in experimental analyses, elaborating on some of the more subtle features which arise from the interplay of the NLO and resummed components of the calculation. We conclude our presentation by comparing predictions from the simulation against a number of Tevatron and LHC jet-production results.

Keywords: Jets, NLO Computations, Hadronic Colliders, QCD

ARXiv EPRINT: 1012.3380 


\section{Contents}

1 Introduction 1

2 Construction of the POWHEG implementation 3

2.1 Next-to-leading order cross sections 4

2.2 Scale choices 4

2.3 Colour assignment in the large- $N_{c}$ limit 4

2.4 Generation cut and suppression factor 6

$\begin{array}{llr}3 & \text { Theoretical analysis and validation } & 7\end{array}$

3.1 NLO cross section 8

3.2 Hardest-emission cross section 8

3.2.1 Parameters for the generation of the samples 10

$\begin{array}{lll}3.2 .2 & \text { Inclusive distributions } & 10\end{array}$

$\begin{array}{lll}3.2 .3 & \text { Jet cross sections with symmetric cuts } & 10\end{array}$

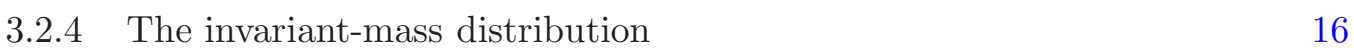

$\begin{array}{lll}3.2 .5 & \text { Jet cross sections with a cut on a single jet } & 17\end{array}$

$\begin{array}{ll}3.2 .6 & \text { Features of the hardest emission }\end{array}$

$\begin{array}{lll}3.2 .7 & \text { Jet structure: the } p_{\mathrm{T}}^{\text {rel }} \text { distribution } & 21\end{array}$

$\begin{array}{lll}3.3 & \text { Parton showering and hadronization } & 22\end{array}$

$\begin{array}{lll}3.3 .1 & \text { Inclusive distributions } & 23\end{array}$

3.3.2 The $R$ dependence of the jet cross section 24

$\begin{array}{lll}3.3 .3 & \text { More exclusive distributions } & 25\end{array}$

4 Phenomenology $\quad 26$

4.1 Multiple-parton interactions in PYTHIA and the POWHEG jet generator 27

$\begin{array}{lll}4.2 & \text { Tevatron results } & 28\end{array}$

4.3 LHC results 31

5 Conclusions $\quad 35$

$\begin{array}{ll}\text { A PYTHIA and HERWIG settings } & 37\end{array}$

\section{Introduction}

Dijet production is by far the most frequently occurring of all hard scattering processes in hadronic collisions, as such it is fundamental that it be thoroughly studied and understood. In keeping with this fact the physics programmes associated with these reactions at hadron colliders are rich and diverse. From a purely experimental perspective, dijet events have an important practical role to play as a tool in various aspects of jet measurement and 
calibration e.g. the determination of the jet energy resolution. Also, from the point of view of QCD, jet pair production in hadronic collisions is particularly interesting in that it is directly dependent on the gluon parton distribution functions at the leading order. More generally, and perhaps more importantly, in providing an abundant source of high momentum transfer events, the dijet production process acts as both a background to, and sensitive probe of, physics beyond the Standard Model.

Indeed, the first measurements and results of new physics searches in this channel, with relatively small amounts of early LHC data, have been publicly documented by the ATLAS and CMS collaborations in recent weeks $[1,2]$. Already these studies have shown perturbative QCD to hold well in new kinematic regimes and extended bounds on an impressive number of new physics models, from composite quarks [3] to $\mathrm{TeV}$ scale string theories $[4,5]$. It is also very clear from these early data, obtained at relatively low energies and luminosities, that in the coming years jet pair production cross sections will be measured with unprecedented precision in the $\mathrm{TeV}$ range.

As with the related Tevatron measurements, the Standard Model predictions used in these analyses are derived from fixed order, next-to-leading-order (NLO) calculations which are corrected for showering, hadronization and underlying event effects estimated using leading-order parton-shower Monte Carlo simulations. Also, in studies such as these, leading-order, leading-log, parton-shower event generators are frequently used in assessing several systematic effects e.g. jet triggering efficiencies and jet energy scale corrections.

Given the significant and wide ranging applications of the dijet production process, and with the LHC now beginning to take data in earnest, the need for refined theoretical modeling is important. Although the level of maturity and attention to detail in current analyses is remarkable, there is still room for improvement. In particular, the way in which the Standard Model prediction is obtained could be made more easily and more coherently through the use of a parton shower simulation consistently including the NLO corrections to jet pair production. An event generator of this nature should also be beneficial in understanding other experimental systematics for which parton shower simulations are relied upon, improving the description of jet profiles through the incorporation of exact, higher-order, real emission matrix elements. Equally, when considered as a background process, all of these higher order QCD corrections will offer markedly better, more robust, predictions than those of the leading-order event generators.

In recent times the construction of such NLO accurate event generators has become viable through the invention of the MC@NLO [6] and POWHEG [7, 8] methods. The effectiveness of these approaches has been demonstrated successfully and studied in some detail through their application to a substantial array of hadron collider processes [9-12]. In this paper we report on our construction and validation of a next-to-leading order parton shower simulation of dijet production according to the POWHEG formalism. To this end we have utilized the public POWHEG BOX package [13], which automates the most complex technical steps of the implementation, essentially reducing it to the task of realising the real, virtual, Born, spin- and colour-correlated Born matrix elements as computer code.

The article is structured as follows. In section 2 we elaborate on the next-to-leading order cross sections underlying the simulation, as employed within the POWHEG BOX, and 
related technical details. In section 3 the validity of the underlying NLO calculation is demonstrated through comparisons with an independent computer code [14] and the implementation of the POWHEG algorithm is checked in a series of non-trivial self-consistency tests. In section 4 we present results from our program in comparison with a number of Tevatron and LHC measurements. Finally, in section 5 we give our conclusions.

\section{Construction of the POWHEG implementation}

As stated in the introduction, we have made use of the POWHEG BOX development framework in building our next-to-leading order parton shower simulation, expediting the process considerably. Essentially, provided with a set of analytic formulae for the real, virtual, spinand colour-correlated Born cross sections, all that is required to produce the corresponding POWHEG simulation are simple computer programs returning their respective values when given a list of particles and their associated momenta. The underlying POWHEG BOX machinery regulates the NLO corrections automatically, using the FKS subtraction formalism [15, 16], and builds the relevant Sudakov form factors internally, combining them to form the POWHEG hardest-emission cross section and, ultimately, an executable to generate the associated events. These single-emission events can then be further evolved to the hadron level by general-purpose parton-shower event generators. In this section we elaborate on the theoretical ingredients and some important technical aspects of the implementation.

In dijet production, only light partons are involved in the Born cross section. The emission of a further light parton has thus one collinear singular region for each Born-level light parton. These regions must be appropriately separated, and this is done (according to the method illustrated in section 4 of ref. [8]) by expressing each real contribution to the cross section (i.e. each matrix element for $2 \rightarrow 3$ scattering) as a sum of contributions, where each contribution is singular only in one collinear region. When computing this contribution, therefore, the singular region itself will be dominant just because of the corresponding singularity. Consider, for example the $q q^{\prime} \rightarrow q q^{\prime} g$ process. The real contribution that is singular when $g$ is collinear to $q$ in the final state, is suppressed in the region where $q^{\prime}$ in the final state becomes collinear to $q^{\prime}$ in the initial state. In other words, the contribution is singular only in the region where the final state $q g$ has the smallest transverse momentum with respect to all other possible collinear pairs. Of course, with this construction, we have left out the possibility that $q g$ in the final state are collinear, and, at the same time, $q^{\prime}$ in the final state is collinear to $q^{\prime}$ in the initial state. This configuration, however, has all particles collinear to the beams, and thus, as we will see, is removed by a generation cut at the Born level. We now stress that with this method the separation of singular regions is not necessarily a sharp one. Thus, in the example adopted above, the singular contribution $q q^{\prime} \rightarrow q q^{\prime} g$ where $g q$ in the final state become collinear, vanishes when $g$ becomes collinear to an initial state parton, but it is non-zero as $g$ approaches this limit. In these non-zero configurations, the transverse momentum of the gluon relative to the beam axis is very small. But the event is assigned a large transverse momentum by POWHEG (i.e. the transverse momentum of the gluon relative to the final-state quark). This transverse momentum is passed to the shower program, that uses it as an upper limit 
for all subsequent emissions, and thus the shower algorithm can generate radiation that is much harder than the $p_{\mathrm{T}}$ of the gluon relative to the beam axis. Of course, the probability to generate configurations like this vanishes with the $p_{\mathrm{T}}$ of the gluon, so that an effective strong ordering of the emissions takes place in POWHEG.

\subsection{Next-to-leading order cross sections}

The next-to-leading order real and virtual matrix elements for dijet production were first computed over twenty years ago [17]. Later a more general approach to the computation of NLO jet observables was considered in ref. [18], wherein one can find, in addition, expressions for the colour-correlated Born cross sections. As input for the POWHEG BOX we have taken the one-loop matrix elements and colour-correlated Born cross sections from the latter publication; the evaluation of the corresponding soft real emission integrals is delegated to the POWHEG BOX. Note that, due to parity conservation, helicity considerations and the fact that the leading-order process comprises of just four massless partons, there are no non-trivial spin correlations among the associated amplitudes. The real cross sections have been built from the concise analytic expressions taken from refs. [17] and [19].

\subsection{Scale choices}

In the POWHEG algorithm, each event is built by first producing what is referred to as an underlying Born configuration, here a QCD $2 \rightarrow 2$ scattering, before proceeding to generate the hardest branching in the event. We have elected to use the $p_{\mathrm{T}}$ of the underlying-Born configuration as the renormalization and factorization scale in obtaining the fixed-order NLO predictions, effectively resumming virtual corrections to the associated $t$-channel gluon propagator. This same scale choice is used in generating the underlying Born kinematics, $\Phi_{B}$, of the POWHEG events (according to the $\bar{B}\left(\Phi_{B}\right)$ function of ref. $[7,8]$ ), while the component of the hardest-emission cross section, responsible for the subsequent generation of the hardest branching kinematics, uses the transverse momentum of the branching, both in the evaluation of the strong coupling constant and the PDFs [8]. Unless otherwise stated, these scale choices are the default ones.

\subsection{Colour assignment in the large- $N_{c}$ limit}

In order to shower and hadronize the hardest-emission events, it is necessary to assign a colour structure to the event, comprised of a number of colour connections: lines charting the flow of colour from one particle to another. To this end, we have adopted the approach proposed in ref. [8], whereby a colour structure is first assigned to the underlying Born configuration probabilistically, according to the relative weight each one contributes to the leading-order cross section in the limit of a large number of colours, $N_{c}$, also known as planar limit. When the hardest branching is generated, its colour structure is assigned by assuming that the colour flow among the mother parton and its two daughters is also trivially planar.

To implement this prescription it is therefore necessary to also compute the Born cross section, piecewise, in terms of the contributions made by each individual colour structure. These component cross sections may be readily computed using large- $N_{c}$ Feynman 

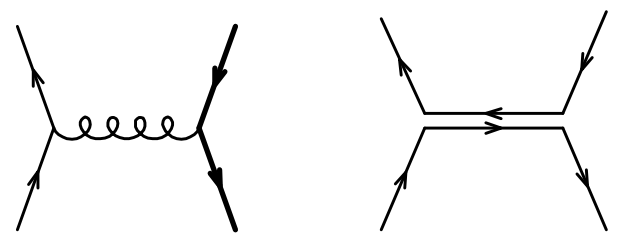

Figure 1. The Feynman diagram for non-identical quark scattering processes, $q \bar{q} q^{\prime} \bar{q}^{\prime}$, with the corresponding planar colour structure depicted on the right.

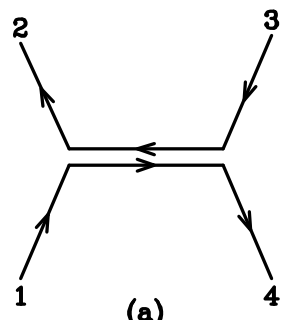

(a)

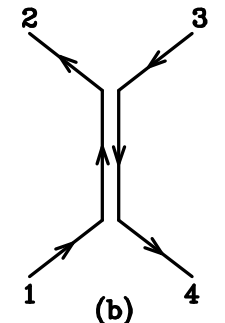

(b)

Figure 2. The two planar colour flows for identical quark scattering processes.
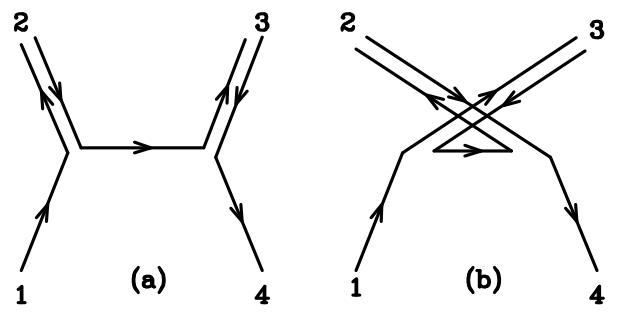

Figure 3. Colour flows in the large $N_{C}$ limit for $q \bar{q} g g$ scattering processes.

rules [20]. For the case at hand, they may also be found in ref. [21]. In the case of a $q \bar{q} q^{\prime} \bar{q}^{\prime}$ amplitude, only one Feynman diagram and one colour structure are involved, with a gluon being exchanged between the $q^{\prime}$ and the $q$ line - in the planar limit, the $q \bar{q}^{\prime}$ and $\bar{q} q^{\prime}$ pairs have opposite colours, as shown in figure 1. For identical quark scattering processes, $q \bar{q} q \bar{q}$, there are only two associated planar colour flows, as shown in figure 2. The corresponding contributions to the leading-order cross section can be easily deduced by comparing the $q \bar{q} q^{\prime} \bar{q}^{\prime}$ and $q \bar{q} q \bar{q}$ squared amplitudes: omitting common factors, they are given by

$$
\left|\mathcal{M}_{(a)}\right|^{2} \propto \frac{s_{14}^{2}+s_{13}^{2}}{s_{12}^{2}}, \quad\left|\mathcal{M}_{(b)}\right|^{2} \propto \frac{s_{12}^{2}+s_{13}^{2}}{s_{14}^{2}},
$$

and the respective colour structures are assigned, probabilistically, on the basis of these values. The two colour flows associated with the $q \bar{q} g g$ process are shown in figure 3 . They are proportional to

$$
\left|\mathcal{M}_{(a)}\right|^{2} \propto\left|\frac{s_{13}}{s_{12}}\right| \frac{s_{12}^{2}+s_{13}^{2}}{s_{14}^{2}}, \quad\left|\mathcal{M}_{(b)}\right|^{2} \propto\left|\frac{s_{12}}{s_{13}}\right| \frac{s_{12}^{2}+s_{13}^{2}}{s_{14}^{2}} .
$$

Thus, the colour structure is chosen with a probability proportional to $s_{13} / s_{12}$ for (a), and $s_{12} / s_{13}$ for (b). Finally, the gggg amplitude has three colour structures, depicted in 

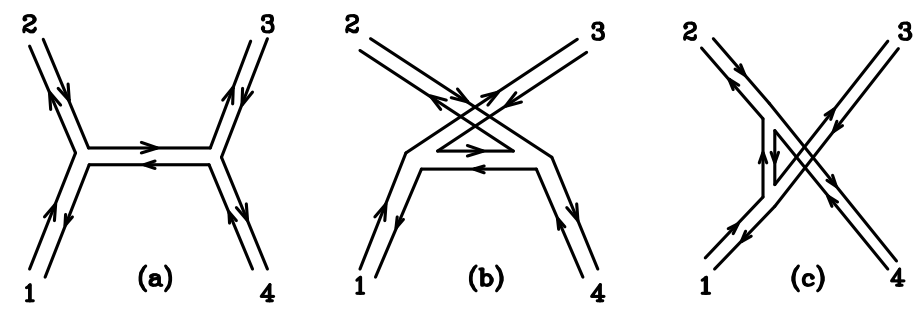

Figure 4. Colour connections for a gggg amplitude.

figure 4. Up to common kinematical factors and physical constants, their contributions to the leading-order cross section are given by

$$
\left|\mathcal{M}_{(a)}\right|^{2} \propto\left(\frac{s_{12}}{s_{14}}+\frac{s_{14}}{s_{12}}+1\right)^{2}, \quad\left|\mathcal{M}_{(b)}\right|^{2} \propto\left(\frac{s_{14}}{s_{13}}+\frac{s_{13}}{s_{14}}+1\right)^{2}, \quad\left|\mathcal{M}_{(c)}\right|^{2} \propto\left(\frac{s_{12}}{s_{13}}+\frac{s_{13}}{s_{12}}+1\right)^{2} .
$$

\subsection{Generation cut and suppression factor}

In the dijet process, as in the $V+j$ process [22], the leading-order contribution to the cross section is itself collinear and soft divergent, mandating that a cut be placed on the transverse momentum of the final-state partons in generating the underlying Born configuration. Since this generation cut is unphysical, it is essential that in studying the output of the simulation, the analysis cuts employed restrict the transverse momenta of the leading jets to always be somewhat larger than it, so as to render any related dependencies negligible. ${ }^{1}$

A further, somewhat related, technical point is that the cross section falls very sharply, as the transverse momenta of the leading jets increases, the rate being crudely proportional to $k_{\mathrm{T}}^{-6}$, hence, generating events with uniform weight generally fails to give a reasonable yield in the high $-k_{\mathrm{T}}$ regions of phase space. One approach to solving this problem is to produce several independent samples of events, using different values of the generation cut in each one, in order to populate all the regions of interest. These samples may then be recombined by weighting events discretely according to the cross section for the sample from which they originated, having taken care to ensure that different samples do not populate the same phase space. Alternatively, the POWHEG BOX is capable of directly generating weighted events samples, as described in ref. [22]. When the weighted event mode is activated (by assigning a positive value to $k_{\mathrm{T}, \text { supp }}$ ), events are no longer distributed according to the differential cross section but rather the differential cross section multiplied by

$$
\mathcal{S}\left(k_{\mathrm{T}}\right)=\left(\frac{k_{\mathrm{T}}^{2}}{k_{\mathrm{T}}^{2}+k_{\mathrm{T}, \text { supp }}^{2}}\right)^{3},
$$

in the case of dijet production. ${ }^{2}$ The generated events now carry a variable weight, equal to the inverse of the suppression factor, $\mathcal{S}\left(k_{\mathrm{T}}\right)$, whose functional form may be changed by

\footnotetext{
${ }^{1}$ For a more involved discussion of this issue see ref. [22].

${ }^{2}$ In the POWHEG BOX, the value of the token bornsuppfact in the input file is assigned to $k_{\mathrm{T}, \text { supp }}$ and the function in eq. (2.4) is implemented in the user-defined subroutine born_suppression.
} 


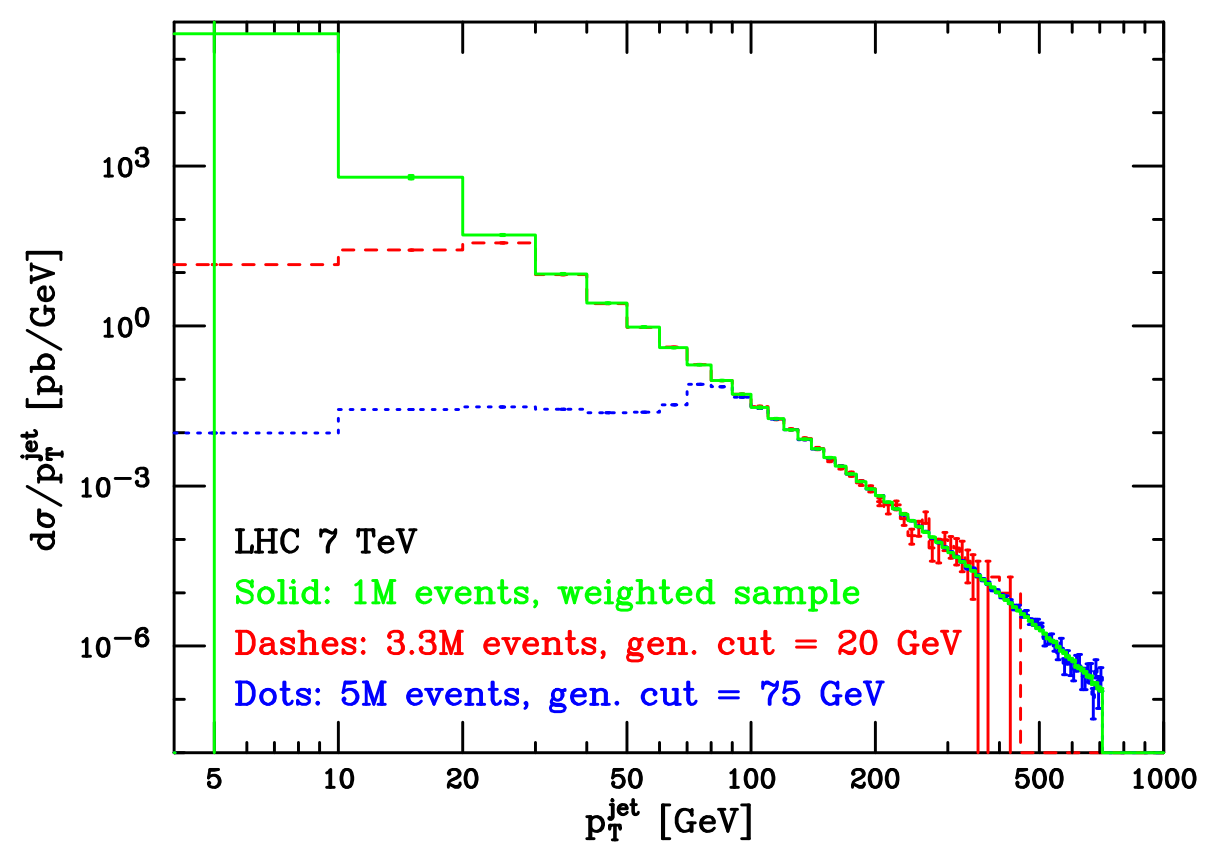

Figure 5. Inclusive differential cross section of the transverse momentum of the leading jet for samples of POWHEG hardest-emission events obtained with different settings, as specified in the figure. We compare two POWHEG unweighted samples for two different generation cuts and a weighted sample with a generation cut at $1 \mathrm{GeV}$. Jets are reconstructed using the CDF midpoint algorithm [23] with $R=0.7$. The weighted sample has been obtained with $k_{\mathrm{T}, \mathrm{supp}}=400 \mathrm{GeV}$.

the user at will, but keeping the same limiting behaviour as $k_{\mathrm{T}} \rightarrow 0$ and $k_{\mathrm{T}} \rightarrow \infty$. The $k_{\mathrm{T}}$ dependence of the suppression factor in eq. (2.4) is such that the generation of low transverse momentum events is relatively damped, while the whole transverse momentum region is nearly uniformly populated up to momenta of the order of $k_{\mathrm{T} \text {,supp }}$. As with the generation cut, a more thorough description of these technicalities may be found in ref. [22].

To illustrate these technical features, in figure 5 we show the jet transverse-momentum spectrum obtained with different generation cuts, and with a weighted sample. The effect of the generation cut on the unweighted sample is negligible above the $p_{\mathrm{T}}$ value where the unweighted sample agrees with the weighted one. Notice also that the weighted sample, in spite of being smaller than the other two, comprising of just 1 million events, populates the region of large transverse momenta well.

\section{Theoretical analysis and validation}

In this section we present results obtained for dijet production in the POWHEG BOX, primarily to audit it and to verify its correctness. This is, however, not quite a simple validation exercise but one very much connected to the phenomenology of jet physics and jet production. In particular we shall consider, in some detail, the interplay of the fixed order component of the calculation and resummation effects. 
Here, as with all results presented in this paper, we have used the CTEQ6M [24] parton distribution functions in generating our predictions. Furthermore, unless otherwise stated we have used the seed-based D0 midpoint cone algorithm, as implemented in the FASTJET package $[25,26]$, with a jet radius of $R=0.7$, an overlap threshold $f=0.5$ and assuming the default values of the minimum jet $E_{\mathrm{T}}$ and minimum jet $E_{\mathrm{T}}$ ratio parameters ( $6 \mathrm{GeV}$ and 0.5 respectively). We have also made some studies using the SISCONE and $k_{\mathrm{T}}$ algorithms [27-29] which we shall discuss in due course.

\subsection{NLO cross section}

The full, regulated, NLO cross section is fundamental in the POWHEG algorithm in that it alone is used to generate the underlying Born configuration, in this case a $2 \rightarrow 2$ QCD process, upon which the whole event is founded. The POWHEG BOX framework has built into it the facility to compute fixed-order NLO distributions for all simulations based upon it. This feature is primarily intended as a diagnostic tool, enabling users to check that the NLO cross section underlying the event generation has been realised correctly within their code. We have made use of this feature to check this delicate component of the simulation, comparing predictions for a wide range of inclusive distributions against the independent parton level program of ref. [14].

In performing these cross checks, we chose to run both programs using a fixed renormalization and factorization scale of $100 \mathrm{GeV}$. Furthermore, in our code, we have used a tiny $k_{\mathrm{T}}$ generation cut of $0.1 \mathrm{GeV}$ and a Born suppression parameter, $k_{\mathrm{T}, \text { supp }}$, of $100 \mathrm{GeV}$. Taking the generation cut to such a small value ensures that the results become insensitive to it, while the Born suppression factor compensates for the sharply falling $k_{\mathrm{T}}$ spectrum such that values of the transverse momentum up to a few hundred $\mathrm{GeV}$ are uniformly sampled. The program of ref. [14] requires a cut on the total transverse energy of the final state which we have stated in the legend of each of the plots. These choices are simply made to ensure a good yield of events in the distributions under consideration, while respecting the jet $E_{\mathrm{T}}$ cuts applied in each case.

In figure 6 we display some results typical of these cross checks. For each plot therein, the two lower plots show the relative difference of the two calculations, $\Delta \sigma / \sigma$, and the difference divided by the statistical error, $\chi$, so defined:

$$
\begin{aligned}
\frac{\Delta \sigma}{\sigma} & =\frac{\sigma_{1}-\sigma_{2}}{\sigma_{2}}, \\
\chi & =\frac{\sigma_{1}-\sigma_{2}}{\sqrt{\delta \sigma_{1}^{2}+\delta \sigma_{2}^{2}}} .
\end{aligned}
$$

All of the distributions we have studied from each code, like those shown in figure 6 , have been found to be fully consistent with one another.

\subsection{Hardest-emission cross section}

Having demonstrated the validity of the underlying NLO cross section, we turn to examine the next phase of the event generation procedure, whereby the hardest branching in the event is generated from the initial $2 \rightarrow 2$ underlying Born configuration. For such a 

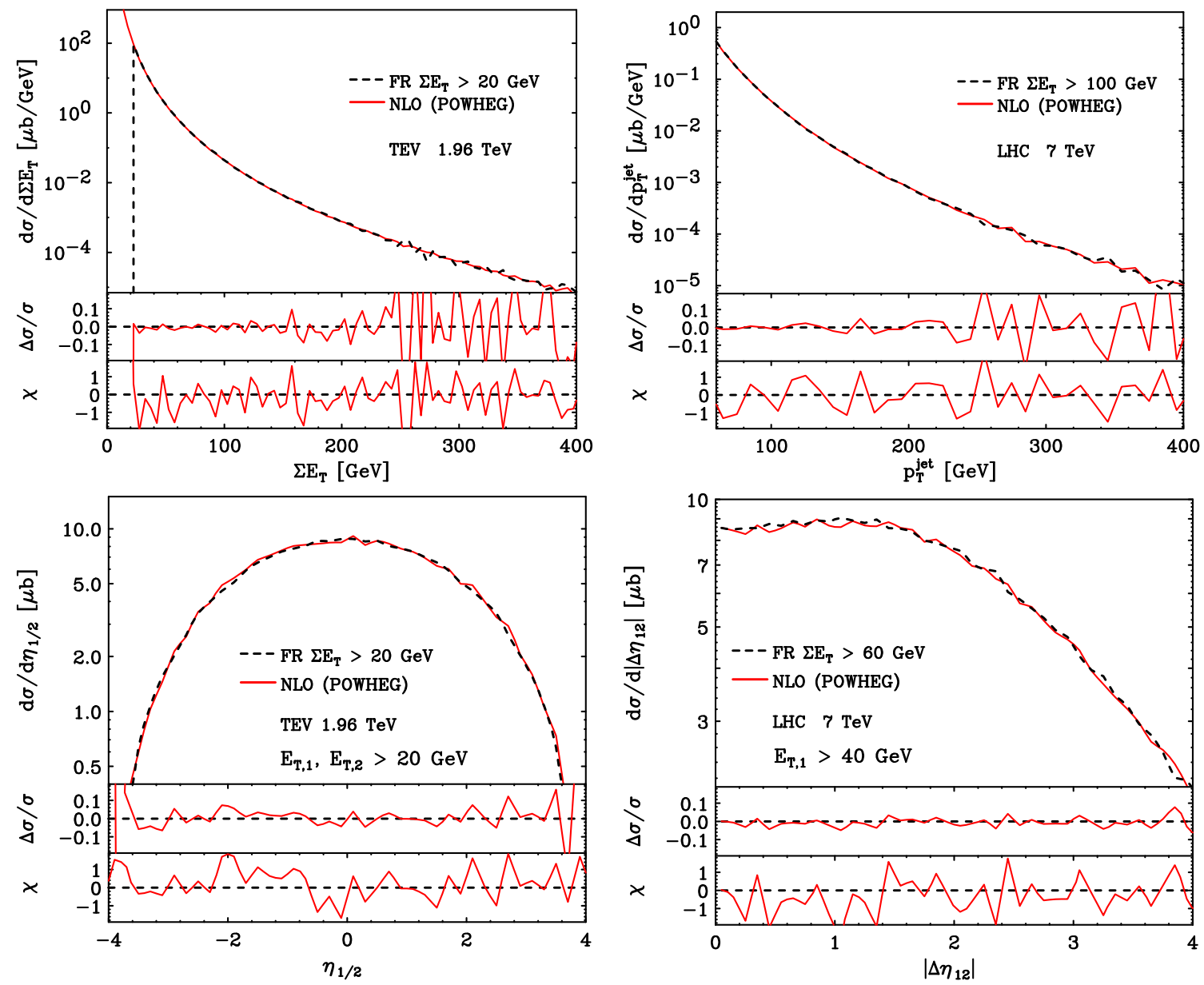

Figure 6. A sample of distributions demonstrating the validity of the underlying fixed-order component of the POWHEG simulation (solid red), comparing it to the NLO calculation implemented in the program of ref. [14] (FR, black dashes). The left-hand column shows cross checks carried out for $1.96 \mathrm{TeV} p \bar{p}$ collisions, while the right-hand column concerns $7 \mathrm{TeV} p p$ collisions. In the upper pair plots we show the total transverse energy spectrum (left) and the inclusive jet $p_{\mathrm{T}}$ spectrum (right). Beneath these are the inclusive pseudorapidity distribution of the two highest transverse momentum jets and that of the pseudorapidity gap between them, for given cuts on their respective transverse energies.

configuration the radiative variables, determining the kinematics of this branching, are distributed according to the product of a Sudakov form factor and the real emission cross section divided by the Born cross section. In this way all orders soft resummation effects are included in the generation of this radiation in the POWHEG hardest-emission cross section [7]. In the following, we wish to assess the impact of such resummation on NLO accurate distributions. To this end, we compare fixed-order NLO predictions, like those in the previous subsection, against those obtained by analysing the hardest-emission events in the Les Houches files prior to their showering with HERWIG or PYTHIA. ${ }^{3}$

\footnotetext{
${ }^{3}$ By setting the testplots token equal to 1 in the input file, two output files are generated that contain these NLO and POWHEG hardest-emission distributions respectively.
} 
In the following analysis, we have used the default scales of section 2.2. Note that using the $p_{\mathrm{T}}$ of the radiation as scale choice for the generation of the hardest emission has no bearing on the distribution of the Born variables $\Phi_{B}$. In fact, given that the same scale is adopted for $\bar{B}\left(\Phi_{B}\right)$ and the fixed-order prediction, the generation of the underlying Born kinematics in the POWHEG simulation and the fixed-order calculation is identical, by construction. This point should be borne in mind throughout this section, in comparing the NLO prediction to those obtained with the POWHEG hardest-emission events.

\subsubsection{Parameters for the generation of the samples}

For the fixed-order computations, we have chosen a generation cut of $1 \mathrm{GeV}$ on the transverse momentum of the underlying Born configuration and a $p_{\mathrm{T}}$ suppression factor parameter $k_{\mathrm{T} \text {,supp }}$ of $50 \mathrm{GeV}$ (see eq. (2.4)). In producing Les Houches event files of hardest-emission events, i.e. events distributed according to the hardest-emission cross section only, we have used a generation cut of $10 \mathrm{GeV}$ and $k_{\mathrm{T} \text {,supp }}=50 \mathrm{GeV}$, for $980 \mathrm{GeV}$ Tevatron beams, while, in simulating LHC events, we have used a $20 \mathrm{GeV}$ generation cut and no Born suppression factor. In the case of the hardest emission events we neglect the effects of negative-weight events, whose presence we have reduced to per mille levels by folding the radiative phase space upon itself according to the technique described in refs. [22, 30].

\subsubsection{Inclusive distributions}

Typically one expects that inclusive observables should exhibit a good level of agreement between the NLO results and those of the POWHEG hardest-emission cross section. More specifically, for quantities that are insensitive to Sudakov effects in the radiation of the third jet, the two sets of results should exhibit deviations no greater than the corresponding expected NNLO corrections. Precisely this behaviour is demonstrated in figure 7 , where the inclusive jet transverse momentum and rapidity spectrum are shown, as given by the same analysis procedure used by the CDF collaboration in ref. [31]. Specifically, we cluster events according to the CDF midpoint cone algorithm, with a jet radius parameter of $R=0.7$, overlapping fraction $f=0.75$ and using the default recombination $E$ scheme, cutting events for which the ratio of the missing transverse energy $\mathbb{E}_{\mathrm{T}}$ to the total transverse energy, $\sum E_{\mathrm{T}}$, fails to satisfy $\mathbb{H}_{\mathrm{T}} / \sqrt{\sum E_{\mathrm{T}}}<\min \left(3+0.0125 \times p_{\mathrm{T}}^{\max }, 6\right)$, all energies expressed in $\mathrm{GeV}$. By analogy with section 3.1, together with the differential cross sections, we plot the relative difference of each POWHEG hardest-emission cross section (solid coloured lines) with respect to the corresponding fixed-order NLO result (black dashes) and the corresponding difference divided by the statistical error, $\chi$, as defined in eqs. (3.1) and (3.2).

\subsubsection{Jet cross sections with symmetric cuts}

We now examine the total cross section for jet production with symmetric cuts on the transverse energy $E_{\mathrm{T}}$ of the two leading jets. In figure 8 we plot the total cross section as a function of $E_{\mathrm{T}, \mathrm{cut}}$, defined to be the cut on the transverse energy of the two highest transverse energy jets: $E_{\mathrm{T}, 1}>E_{\mathrm{T}, \mathrm{cut}}, E_{\mathrm{T}, 2}>E_{\mathrm{T}, \mathrm{cut}}$. For both plots, obtained at Tevatron and LHC energies respectively, we show the fixed-order NLO prediction as a dashed black line, with the corresponding POWHEG hardest-emission cross section, in solid red. At first 

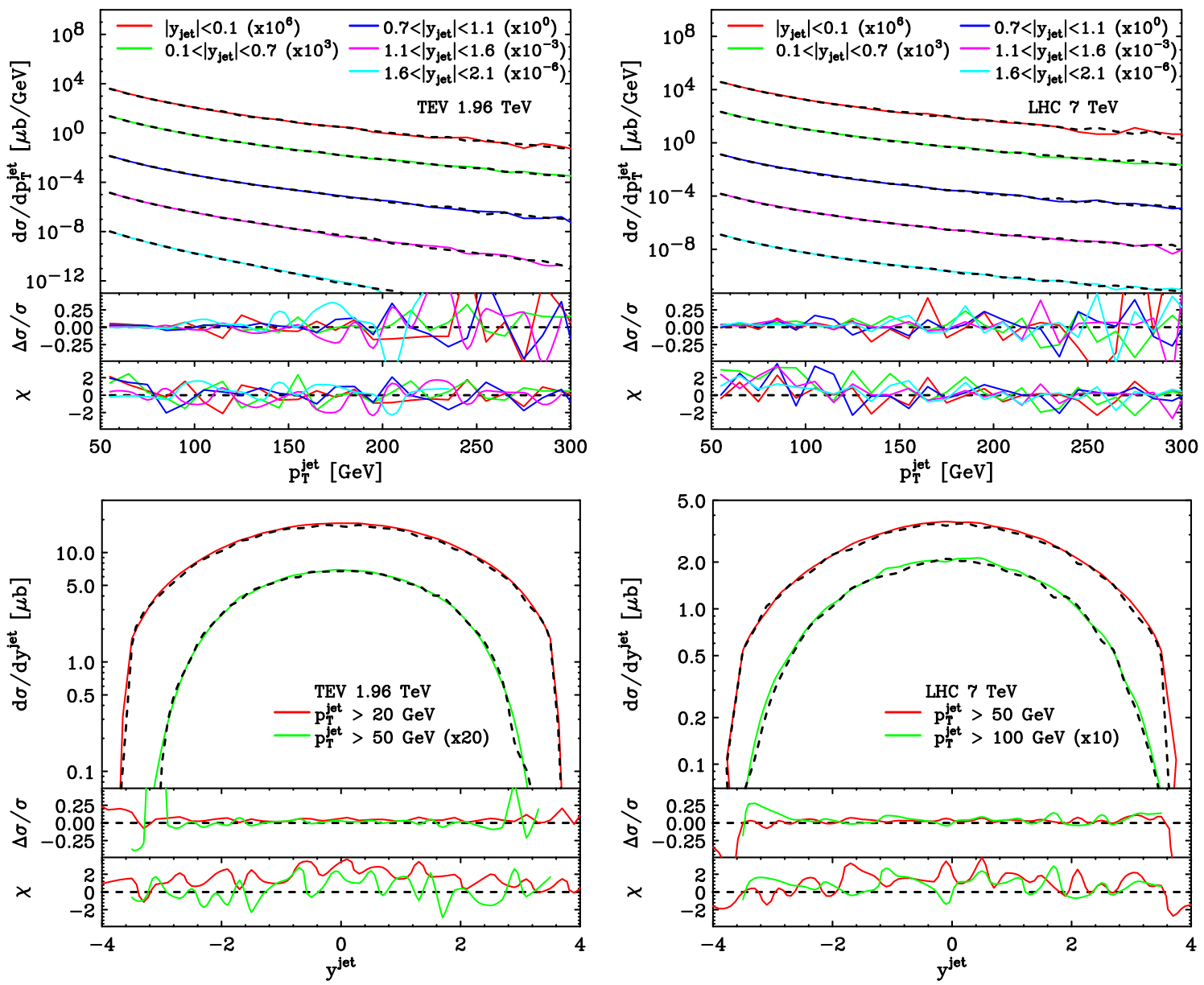

Figure 7. A comparison of the POWHEG results, prior to showering (solid coloured lines), to the corresponding fixed-order NLO predictions (black dashes) of the inclusive jet transverse-momentum and rapidity distributions. Coloured curves in the upper two plots are drawn, from top to bottom, in order of increasing rapidity, while in the lower plots the result obtained with the greater of the two transverse-momentum cuts is lowermost.

sight the disagreement between the fixed-order and the POWHEG results may not seem so remarkable. However, a quick look at the distributions of $\Delta \sigma / \sigma$ and $\chi$, in the lower panels, reveals that the prediction of the resummed NLO prediction is around a factor of two higher than that of the fixed-order NLO calculation, when $E_{\mathrm{T}, \mathrm{cut}}$ tends to small values. This large discrepancy is alarming, particularly given that there is certainly nothing untoward about these cuts from the point of view of infrared safety. However, instabilities of NLO jet production cross sections in the presence of symmetric cuts on jet transverse energies have been noted and studied in the past, in lepton-hadron $[14,32]$ and hadron-hadron collisions [33].

In order to reconcile the predictions of the NLO and POWHEG hardest emission cross sections we have carried out a similar analysis to that performed in ref. [14] in the context of two-jet photoproduction in lepton-hadron collisions, the results of which are displayed in figure 9. Here we have considered the total cross section as a function of $\Delta$ which parametrises the degree to which the cuts on the leading- and next-to-leading- $E_{\mathrm{T}}$ jets are 

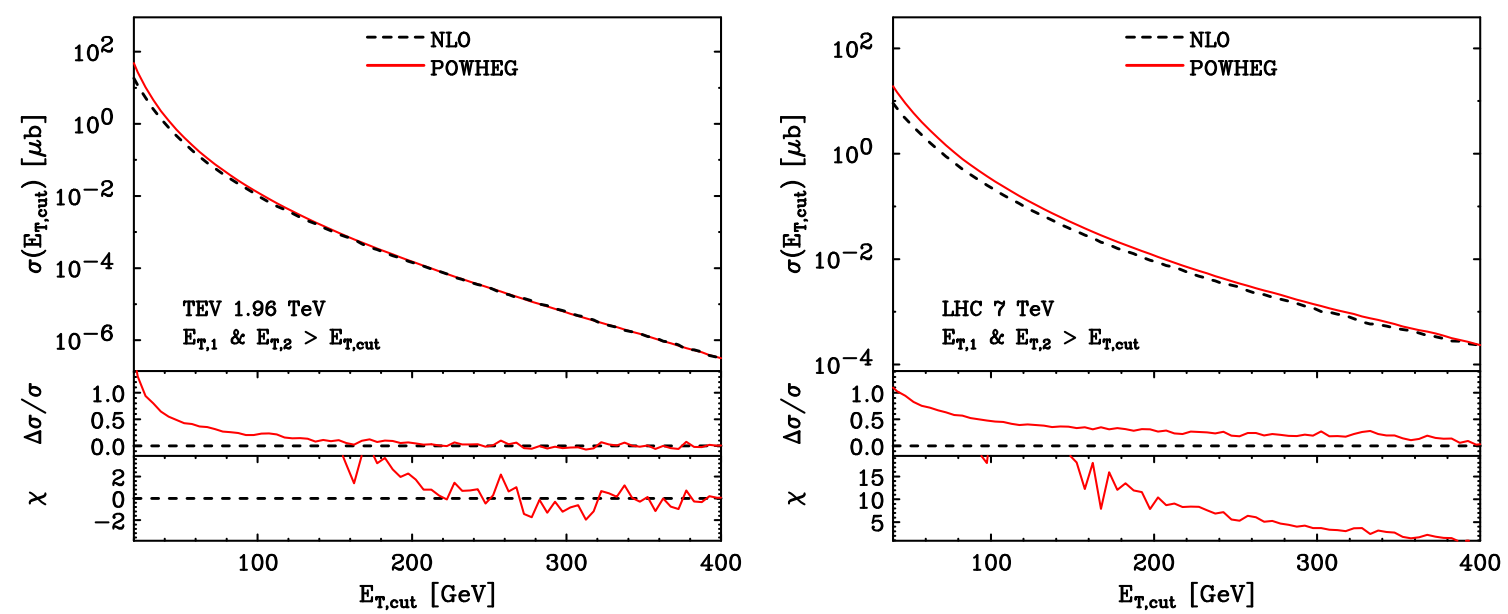

Figure 8. Predictions for the fixed-order NLO cross sections to the analogous POWHEG hardestemission one, for symmetric cuts on the transverse energies of both the highest and second highest $E_{\mathrm{T}}$ jets, at the Tevatron and LHC, in the left- and right-hand plots respectively.

asymmetric: $E_{\mathrm{T}, 1}>\Delta+E_{\mathrm{T}, \mathrm{cut}}, E_{\mathrm{T}, 2}>E_{\mathrm{T}, \mathrm{cut}}$, the limit $\Delta \rightarrow 0$ therefore corresponding to the case of symmetric cuts. In the case of the fixed-order predictions, for LHC and Tevatron collider configurations, and for all studied values of $E_{\mathrm{T}, \mathrm{cut}}$, we observe the same behaviour as in ref. [14]. In particular, counter to one's expectations based on simple phase-space considerations, we find that the cross section is not monotonically increasing with decreasing $\Delta$ but rather it rises gradually to a peak as $\Delta$ tends to small values, before falling sharply as $\Delta \rightarrow 0$. This is in contrast to the POWHEG prediction which simply continues to rise.

In the case of two-jet photoproduction the nature of this peculiar turn over in the NLO distribution was explained by the authors of ref. [14] as being due to the emergence of large, dominant, logarithmic terms of the form $-\Delta \log \Delta$ in the real part of the NLO cross section. This functional dependence on $\Delta$ is plainly manifest around the $\Delta \rightarrow 0$ region in our fixed-order predictions. Although our study concerns dijet hadroproduction, the explanation advocated in ref. [14] readily applies here too without modification, since exchanging the initial-state photon for an initial-state parton does not qualitatively affect the leading collinear singular behaviour of the real cross section.

It is also stated in ref. [14] that the fall in the fixed order predictions generated by the $-\Delta \log \Delta$ term is symptomatic of the truncation of perturbative series at NLO, and that the resummation of higher-order soft-virtual corrections will oppose this effect. In ref. [33], such resummation has been performed, and the behaviour of the distributions found there is very similar to the one in figure 9. In POWHEG, such resummation is implicit in the hardest-emission cross section, via the Sudakov form factor, which acts precisely in this way, inhibiting the same soft and collinear emissions which give rise to the $-\Delta \log \Delta$ terms. Having suppressed these spurious strong dynamical contributions appropriately, the POWHEG predictions, shown as coloured lines in figure 9, do not follow the same trends set by the fixed-order predictions, but simply decrease along with the available phase space. 

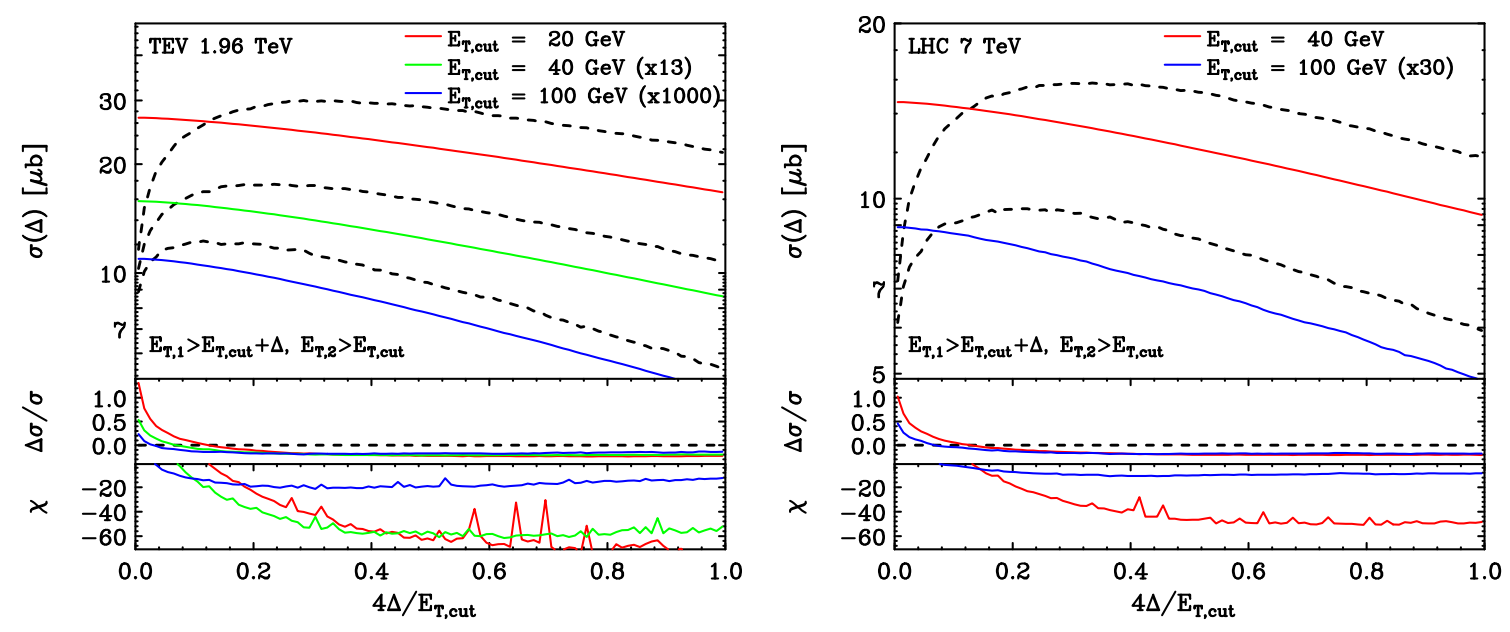

Figure 9. Predictions for fixed-order NLO cross sections (black dashes) compared with those of the POWHEG hardest-emission cross section (coloured solid lines), as a function of the transverse energy cut parameters $\Delta$ and $E_{\mathrm{T}, \text { cut }}$, defined by the relations: $E_{\mathrm{T}, 1}>E_{\mathrm{T}, \text { cut }}+\Delta, E_{\mathrm{T}, 2}>E_{\mathrm{T}, \text { cut }}$, with $E_{\mathrm{T}, 1}$ and $E_{\mathrm{T}, 2}$ being the transverse energies of the leading and next-to-leading jets, ordered according to transverse momentum. Results for the Tevatron on the left, and for the LHC on the right. Coloured lines are ordered from top to bottom with increasing $E_{\mathrm{T}, \mathrm{cut}}$.

The rôle of the underlying Born configuration. In POWHEG, the division of the realemission phase space into that of the underlying Born configuration and that of the hardest branching lends itself to an alternative, more mechanical, understanding of these effects. In particular, it is instructive to consider a posteriori how events passing and/or failing the symmetric cuts have originated, i.e. the associated $2 \rightarrow 2$ underlying Born kinematics. For the case at hand, since transverse energy cuts are applied to the two leading jets, we are especially interested to know if and how events migrate across the cut depending on the transverse momentum of their underlying Born kinematics. We stress that this transverse momentum, as with all quantities deriving from the underlying Born kinematics, $\Phi_{B}$, is infrared safe; this follows directly from the definition of the mappings used to relate underlying Born kinematics to those of real emission configurations. ${ }^{4}$

Accordingly, in figure 10 we show four distributions of the underlying Born transverse momentum. In the first plot on the upper left-hand side we show predictions for this quantity as given by NLO QCD (black dashes) and the hardest-emission cross section (red solid) with no analysis cuts applied. As stated earlier, these two distributions are the same by construction, the only difference being the use of a higher generation cut on the $p_{\mathrm{T}}$ of the underlying Born kinematics in the latter case $(10 \mathrm{GeV}$ as opposed to $1 \mathrm{GeV})$. In the upper right-hand corner we plot the same quantity but now only for events which pass the symmetric cuts of $40 \mathrm{GeV}$ in $p_{\mathrm{T}}$ on the final-state jets. Above the cut, the distribution falls rapidly, as in the first plot where no cuts were applied. Conversely, between 0 and $40 \mathrm{GeV}$ the distribution increases. Below $p_{\mathrm{T}}=40 \mathrm{GeV}$ the distribution is populated exclusively by

\footnotetext{
${ }^{4}$ In case the reader requires further clarification as to whether the underlying Born transverse momentum is a truly physical quantity, we point out that further discussion and assurance is given in the second half of section 3.2.5.
} 

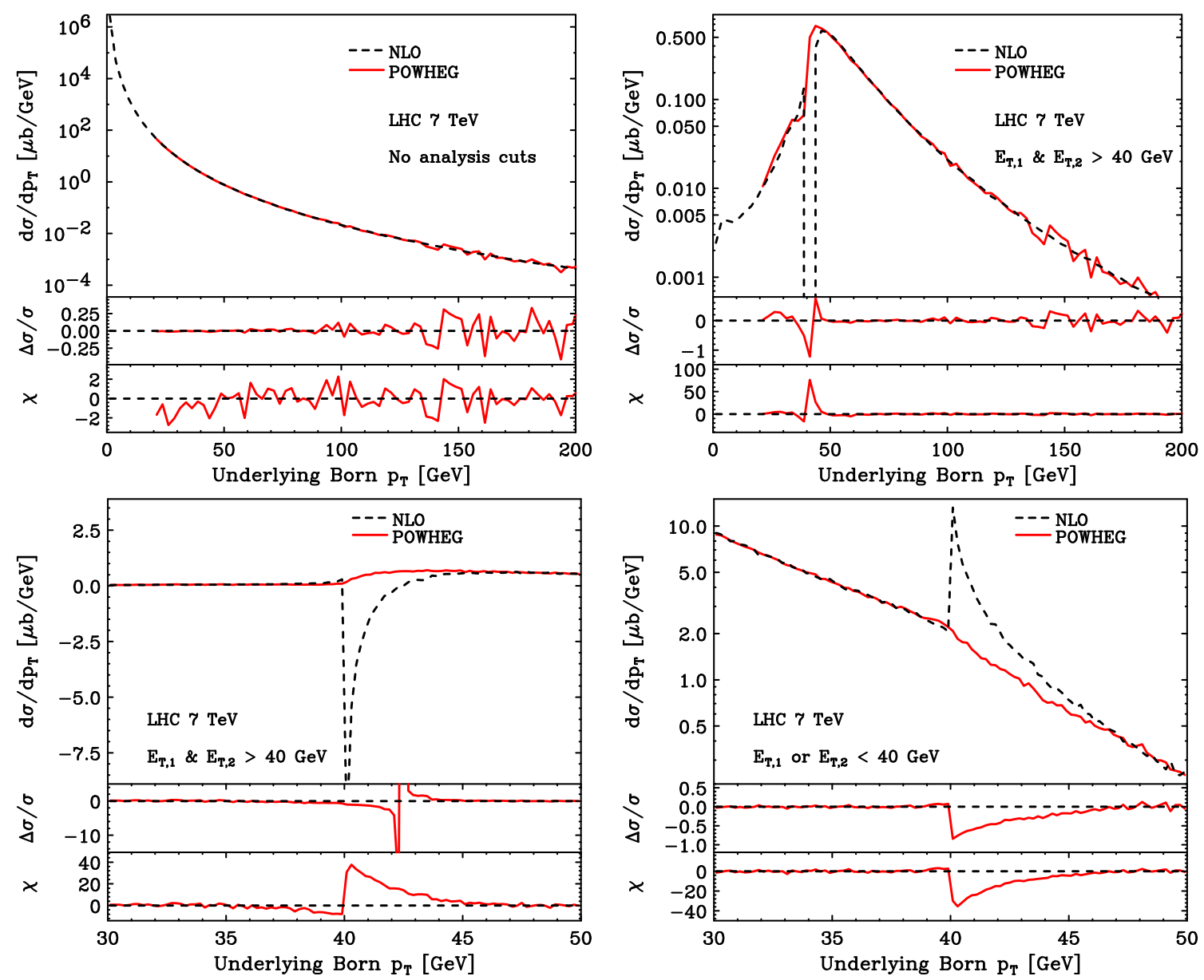

Figure 10. The transverse-momentum spectra for the underlying Born configuration having applied symmetric cuts on the transverse energies of the jets in 2- and 3-parton events and, in the case of the fixed-order NLO results, to the counter-events too. The results from POWHEG hardest-emission events are shown in solid red, while the corresponding fixed-order NLO predictions are drawn as dashed black lines.

events initially comprised of two partons with $p_{\mathrm{T}}<40 \mathrm{GeV}$, which radiate to yield events containing two jets with $E_{\mathrm{T}}>40 \mathrm{GeV}$ : the rise in the distribution towards $40 \mathrm{GeV}$ simply reflects the fact that less radiative phase space is required to produce an event passing the cut when the underlying Born configuration is closer to passing the cut itself.

It is quite remarkable that one can see very good agreement between these NLO and hardest-emission cross sections, at the level of about $10 \%$, for almost all of the range in $p_{\mathrm{T}}$, yet from figure 8 we know that the NLO cross section for these cuts is around half that of the POWHEG hardest-emission cross section. One may think that the extra phase space available in the case of the fixed-order calculation, due to having used a $1 \mathrm{GeV}$ instead of a $10 \mathrm{GeV}$ generation cut in the underlying Born $p_{\mathrm{T}}$ may cause the discrepancy. However, this could only explain an excess of the NLO result, whereas it is the hardest-emission cross section which is the greater of the two. Moreover, by closer inspection of the results, one can see that the additional region populated by the fixed-order calculation contributes only $1.7 \%$ of the total cross section. 
In fact, the factor of two deficit in the NLO calculation with respect to that of the hardest-emission cross section can be wholly attributed to the apparently empty bin between 40 and $42.5 \mathrm{GeV}$. A close up of this $p_{\mathrm{T}}$ region can be seen in the lower left-hand plot and the same distribution, in the same region, can be seen for events failing the symmetric cuts in the lower right-hand plot. The unstable nature of the fixed-order calculation is very clear as a discontinuity in the first derivative of the $p_{\mathrm{T}}$ distribution of the underlying Born kinematics. Moreover, we can see that in the spurious bin in the NLO distribution, $40.0<p_{\mathrm{T}}<42.5 \mathrm{GeV}$, the negative-weight counter-events, having $2 \rightarrow 2$ kinematics specified by $\Phi_{B}$, pass the cut by construction, while the corresponding three-body real emission events, constructed from exactly the same $\Phi_{B}$ and additional radiative variables $\Phi_{R}$, migrate below the cut (bottom right plot). The fact that the NLO cross section is seen to be negative in the region just above $40 \mathrm{GeV}$, together with the correspondence in the size of the excess (deficit) in the NLO events failing (passing) the cut, is an unmistakable sign that this mechanism is in effect. By contrast, in the POWHEG case, one has uniquely positiveweighted events as opposed to events and counter-events, moreover, the generation of the soft and collinear emissions, which cause the real radiation events to fail the jet $E_{\mathrm{T}}$ cut by only a small amount, but in large numbers, is Sudakov suppressed. Hence, the POWHEG prediction exhibits no such anomalous behaviour, instead it rises to a smooth, rounded, peak which falls away to the left, just before the $40 \mathrm{GeV}$ mark is reached, very similar to the full resummed result [33].

In summary, the NLO dijet cross section, for the case of symmetric cuts on the leading jets, while being formally infrared safe, exhibits pathological behaviour e.g. the fall in the total cross section encountered as $\Delta \rightarrow 0$ in figure 9 and the discontinuous distributions which it predicts for the transverse momentum of the underlying Born configurations in figure 10. These eccentricities are the result of an acute sensitivity of the cross section to soft emission effects, which are not properly handled in the fixed-order computation. The mechanism behind this sensitivity can be clearly understood in terms of soft, three-body, real-radiation events migrating below the cuts while their corresponding two-body counterevents remain above it, in the NLO case (figure 10). The inclusion of the proper Sudakov suppression for this soft radiation in POWHEG largely fixes this abnormal behaviour, leading to more physical predictions.

In parting we wish to make two further qualifying remarks regarding these conclusions. Firstly, as one might expect, developing the POWHEG events more fully, by including the subsequent parton showering and hadronization, depletes the jet transverse energies through emission of out-of-cone radiation, lessening the level of disagreement. However, for the distribution shown in figure 8 , the showered and unshowered predictions always agree to within $15 \%$. Secondly, we note that it may be tempting to think that the anomalous behaviour shown here by the fixed-order results may somehow arise through a deficiency, e.g. a lack infrared safety, in the jet algorithm; yet we have repeated this exercise using the SISCONE $(R=0.7, f=0.5)$ and inclusive $k_{\mathrm{T}}$ jet algorithms $(R=0.7, E$-scheme) finding qualitatively the same features: a large deficit of the NLO prediction with respect to the hardest-emission cross section, by at least a factor of two in both cases, and a solitary, highly negative bin entry at $40.0<p_{\mathrm{T}}<42.5 \mathrm{GeV}$ in the transverse momentum spectrum of the underlying Born configuration. 

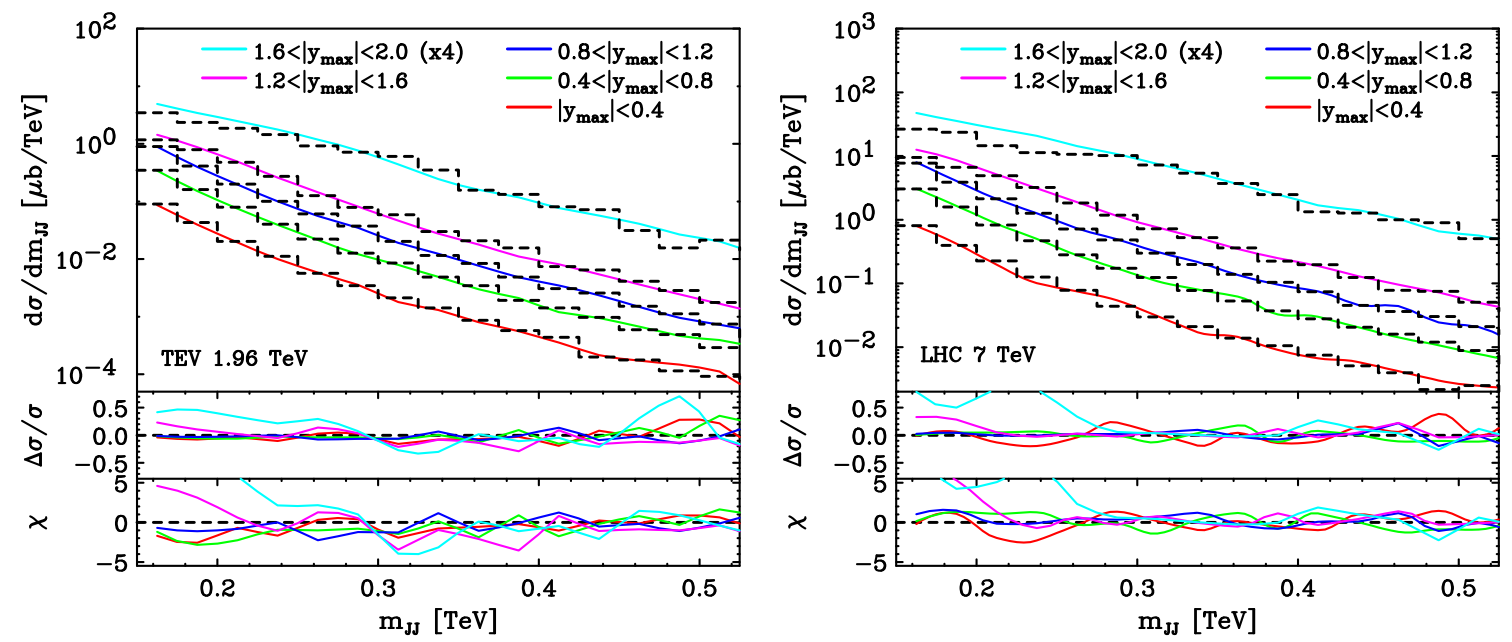

Figure 11. In coloured lines the POWHEG hardest-emission distributions for the dijet invariant mass (prior to parton showering) with their analogous fixed-order NLO counterparts overlaid (black dashes). Coloured lines are ordered from bottom to top in increasing rapidity intervals.

\subsubsection{The invariant-mass distribution}

As an interesting example of the effect of symmetric cuts on experimentally-measurable distributions, we present in figure 11 the dijet invariant-mass distributions, resulting from analysis of the POWHEG hardest-emission events (coloured lines), superimposed on the corresponding fixed-order predictions (black dashes), for both Tevatron and LHC collider configurations. In keeping with the analysis of ref. [34], we impose a symmetric $40 \mathrm{GeV}$ transverse-momentum cut on the two leading $p_{\mathrm{T}}$ jets and we constrain the rapidity of the most forward of these, $|y|=\max \left(\left|y_{1}\right|,\left|y_{2}\right|\right)$, to lie within one of several bins (shown inset). It is clear that, for a given value of dijet invariant mass, these selection criteria favour events in which the rapidities of the two highest $p_{\mathrm{T}}$ jets have opposite sign, their absolute value lying in the same bin. The rapid fall in the cross section as the invariant mass increases is, of course, expected from simple phase space considerations, while the growth with increasing $|y|$ reflects the fact that, at fixed invariant mass, higher jet rapidities correspond to smaller angle, lower transverse-momentum scatterings, which yield larger cross sections.

As with the case of the inclusive jet transverse-momentum spectrum, here we find that the level of agreement between the POWHEG and NLO predictions is generally very good, with the exception of the highest rapidity bins in the low invariant mass region, where the former is found to exhibit a $50 \%$ excess. In light of our previous discussion on the effects of symmetric jet $E_{\mathrm{T}}$ cuts this excess is readily explainable. Note that the invariant mass spectrum shown here begins at $160 \mathrm{GeV}$, corresponding to the production of pairs of jets in the central region with transverse energies of around $80 \mathrm{GeV}$. However, should the leading jets be produced at high rapidities, the cross section will be dominated by pairs of back-to-back jets with smaller $p_{\mathrm{T}}$ and a larger rapidity separation. It is easy to check that for rapidities around 1.6 the transverse momentum of the jets in the back-toback configuration approaches $40 \mathrm{GeV}$. The effects of the symmetric $p_{\mathrm{T}}$ cuts then play an 

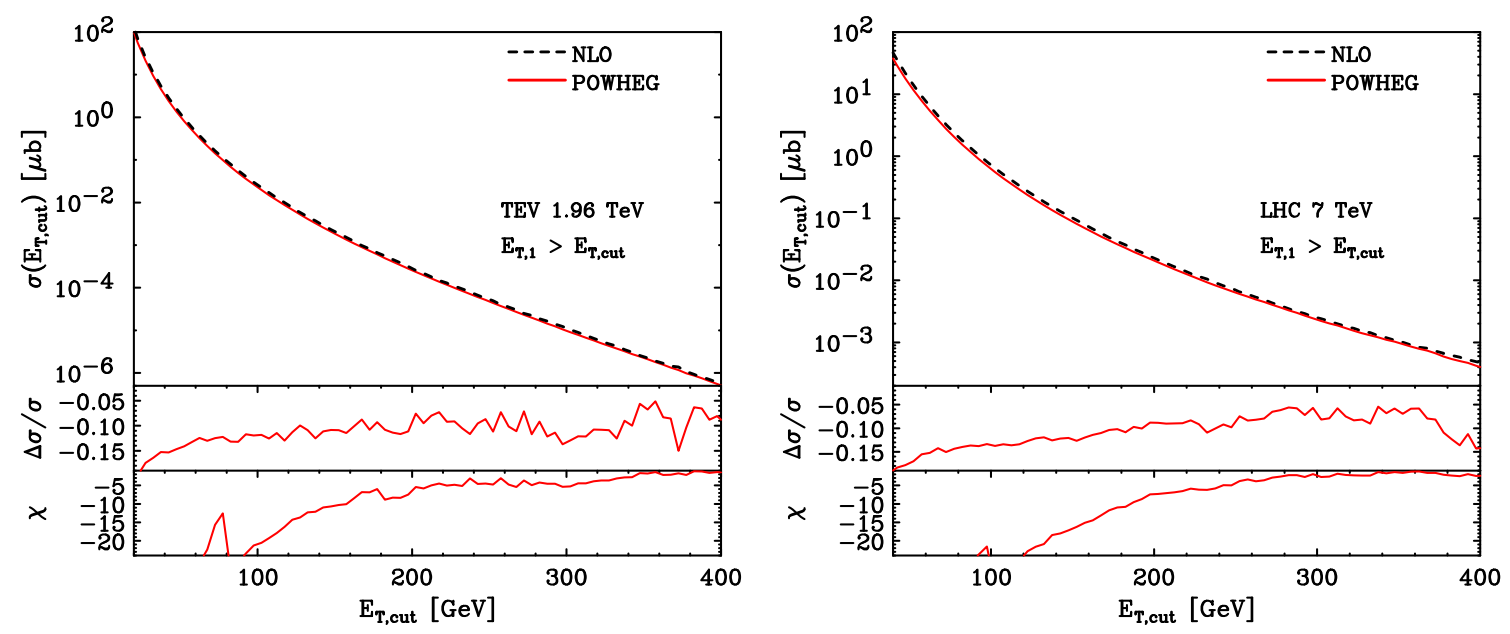

Figure 12. As in figure 8, here we compare fixed-order NLO cross sections (black dashes) to the corresponding POWHEG hardest-emission cross section (solid red), however, in this case we have applied the jet transverse-energy cut to the highest- $E_{\mathrm{T}}$ jet alone.

increasingly significant role and so the previously discussed POWHEG excess over fixed-order predictions for such cuts becomes visible.

\subsubsection{Jet cross sections with a cut on a single jet}

As stated previously, the frailties of NLO calculations for dijet production with symmetric cuts were first noted quite some time ago. This has led to the general consensus that asymmetric cuts on jet transverse energies should be used in theoretical studies and in confronting data with next-to-leading order QCD predictions. It is therefore of practical and academic interest to repeat the analysis of section 3.2.3 for such cuts.

In figure 12 we compare the POWHEG hardest-emission cross section against fixed-order predictions as a function of $E_{\mathrm{T}, \mathrm{cut}}$, here redefined to be a transverse energy cut on the highest transverse-energy jet alone: $E_{\mathrm{T}, 1}>E_{\mathrm{T}, \mathrm{cut}}$. This is a more inclusive quantity with respect to the case of symmetric cuts (but still less inclusive with respect to the inclusive jet cross section). We then note, with interest, an excess of the fixed-order prediction over that of the resummed calculation, in the region of $10-20 \%$ across most of the range in $E_{\mathrm{T}, \mathrm{cut}}$. This level of disagreement may not be particularly bothersome in the context of a differential distribution in some infrared safe quantity, and one can expect that parton showering and hadronization may combine to alter this picture by a similar amount. However, these differences occur at the level of the total inclusive cross section subject to what are effectively maximally asymmetric cuts, $E_{\mathrm{T}, 1}>E_{\mathrm{T}, \mathrm{cut}}$, hence, they must be investigated.

The rôle of the underlying Born configuration. Recall that the underlying Born kinematics, $\Phi_{B}$, is generated by the exact same mechanism in the fixed order and POWHEG predictions, thus any disparity between the two must follow from the way in which radiation is generated from this initial $2 \rightarrow 2$ configuration. Hence, here again, we choose to interrogate the events passing or failing the jet $E_{\mathrm{T}}$ cut regarding the transverse momentum of their underlying Born configuration. 

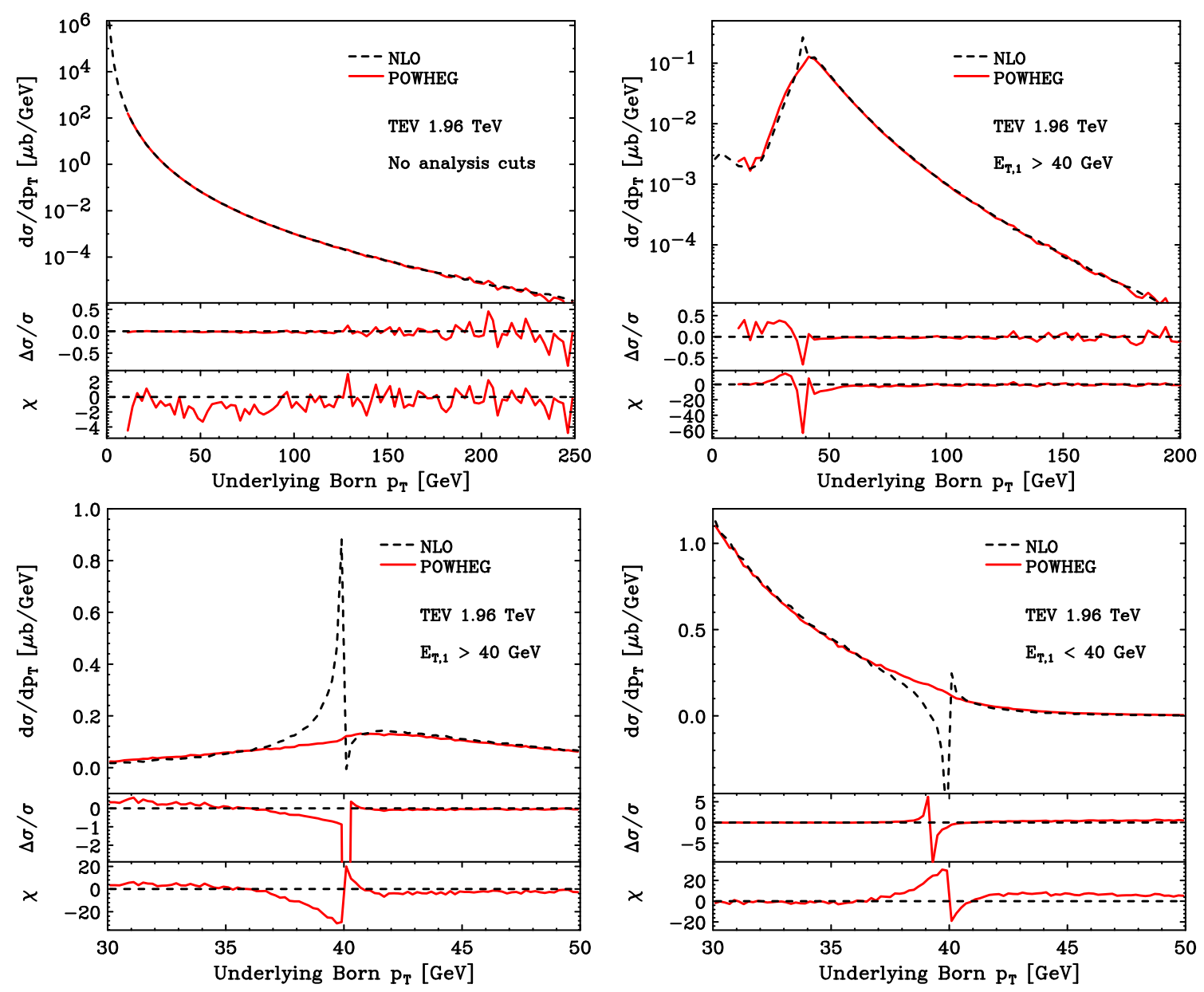

Figure 13. The transverse-momentum spectra for the underlying Born configuration having applied a cut only on the highest $E_{\mathrm{T}}$ jet. The results from POWHEG hardest-emission events are shown in solid red, while the corresponding fixed-order NLO predictions are drawn as dashed black lines.

In figure 13 we plot the same set of distributions as in figure 10 for the case of a cut on the $E_{\mathrm{T}}$ of the leading jet alone. The resulting picture reveals many of the same features seen in the analogous symmetric $E_{\mathrm{T}}$ jet cut analysis. The integrated bin contents confirms that the prediction from the hardest-emission cross section is $15 \%$ below that of the fixed-order result, with only $1 \%$ of this due to the unpopulated region below the $10 \mathrm{GeV}$ generation cut in POWHEG. The remaining excess is attributed to a lone spike in the fixed order distribution, in the bin at $37.5<p_{\mathrm{T}}<40.0 \mathrm{GeV}$, in the plot shown in the upper right-hand corner of figure 13. This region of the spectrum is shown magnified in the bottom left corner of the same figure, with the complementary distribution for events failing the $E_{\mathrm{T}}>40 \mathrm{GeV}$ cut shown alongside it.

Remarkably, the fixed-order distribution is found to be prone to pathological behaviour, being discontinuous in its first derivative at $p_{\mathrm{T}}=40 \mathrm{GeV}$, while the corresponding POWHEG result is smooth and physical. The fixed-order distributions show how events, in which the $p_{\mathrm{T}}$ of the underlying Born kinematics is below, yet close to, the cut, migrate above it, 
through the emission of radiation. The related, negative weight, two-body counter-events have the kinematics of the underlying Born configuration, hence, these remain below the cut where they give rise to a negative cross section for events failing it in the region near $40 \mathrm{GeV}$. The fact that the distribution diverges as the cut is approached clearly indicates that the majority of the upward migration is due to soft radiation. Of course, the rate of these soft emissions in the fixed order calculation is erroneous, containing no Sudakov suppression factor, unlike the POWHEG hardest-emission cross section. This explains why a similar diverging and discontinuous distribution is not present in its associated predictions.

Once again, we may conclude here that the predictions of the hardest-emission cross section offer a considerably improved description with respect to their fixed-order counter parts. Nevertheless, unlike in the case of symmetric jet $E_{\mathrm{T}}$ cuts, this point should not be overemphasised here, since the differences which led us to examine the calculations, as in figure 13, were only at the level of 10-15\%. Parton showering and hadronization, in the case of POWHEG, and non-perturbative correction factors, in the case of fixed-order computations, contribute to shift these predictions by similar degrees.

Before continuing we wish to allay any concerns which the reader may have as to the physical nature of the analyses depicted in figures 10 and 13. In particular, one might well wonder to what extent the transverse momentum of the underlying Born configuration is a physical quantity and so query the validity of our explanations. Taking into account the parton showering, hadronization and underlying event effects that occur in reality, it is certainly the case that this quantity is not experimentally measurable. However, from the point of view of the three-body events originating from the NLO and POWHEG hardest-emission cross sections, one can construct effectively a jet algorithm, based on the POWHEG phase-space factorisation and mappings, which clusters them back to a two-body underlying Born configuration.

In any case, to quell any doubts that may have arisen, we point out that we have repeated the analysis surrounding these figures plotting, instead of the underlying Born $p_{\mathrm{T}}$, the average transverse momentum of a pair of jets, $\left\langle p_{\mathrm{T}}\right\rangle$, obtained by applying the exclusive $k_{\mathrm{T}}$ jet algorithm, demanding it returns always just two jets, using the $E_{\mathrm{T}}$ recombination scheme (rather than the default $E$ scheme). This effectively projects the three-body realemission kinematics to a massless two-body configuration, as in the underlying Born $\Phi_{B}$. Clearly this quantity is very closely related to the underlying Born transverse momentum, converging to it in the limit of soft and collinear emissions. In so doing, we see essentially the same distributions, with the same structure and features, as in figures 8-13. In particular we note the continuing presence of the discontinuities in the fixed-order predictions. At a quantitative level, the fact that we used the $k_{\mathrm{T}}$ jet clustering algorithm to define all jets in the analysis changes things but not in such a way as to alter our conclusions. Whereas in the symmetric $E_{\mathrm{T}}$ cut case, with the D0 midpoint cone algorithm, the cross section for $E_{\mathrm{T}, 1}>40 \mathrm{GeV}$ and $E_{\mathrm{T}, 2}>40 \mathrm{GeV}$ revealed a deficit of $105 \%$ with respect to the POWHEG prediction, falling to $3 \%$ on neglecting the large negative bin at $40.0<p_{\mathrm{T}}<42.5 \mathrm{GeV}$ in the underlying Born $p_{\mathrm{T}}$ distribution, when using the $k_{\mathrm{T}}$ algorithm, with $R=0.7$, the deficit was instead $250 \%$ reducing to $22 \%$ on omitting the same bin in the $\left\langle p_{\mathrm{T}}\right\rangle$ distribution. In the case of the lone $E_{\mathrm{T}, 1}$ cut scenario, the fixed-order prediction with the D0 midpoint cone algorithm 

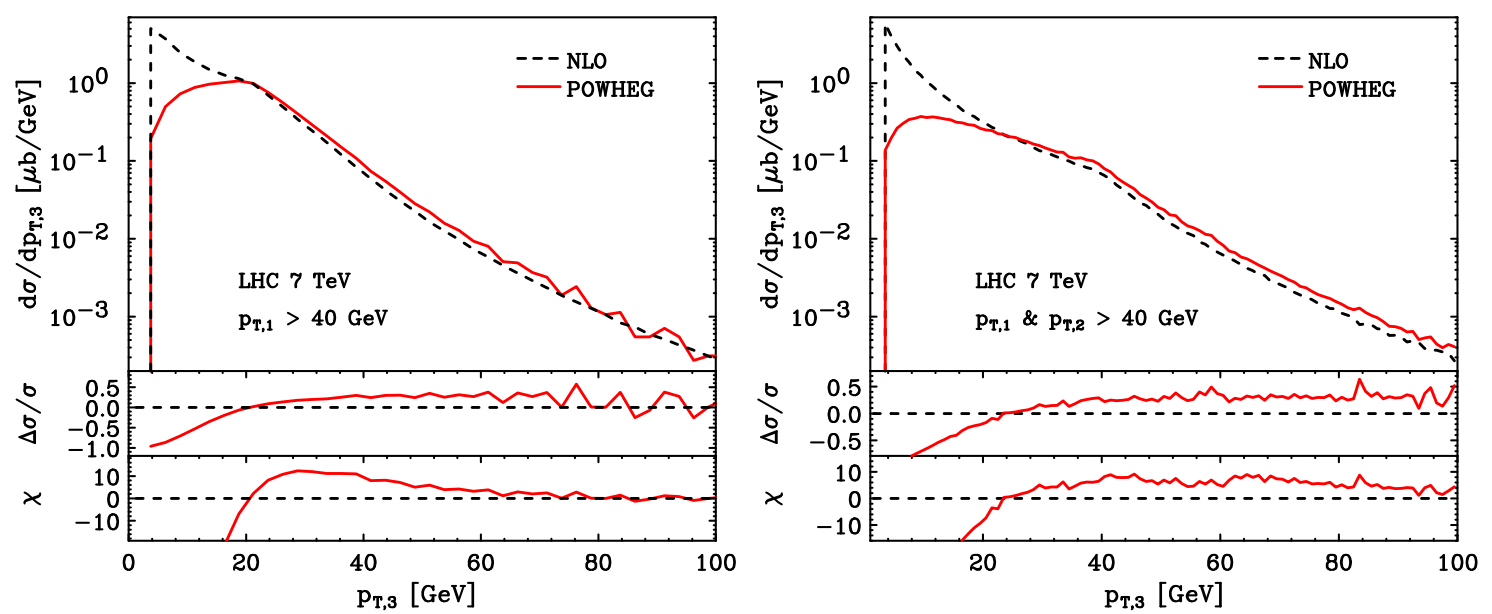

Figure 14. The transverse-momentum spectrum of the third hardest jet as given by the NLO cross section (black dashes) and POWHEG without parton showering (solid red). In the left-hand plot we have applied a transverse-momentum cut on the hardest (highest $p_{\mathrm{T}}$ ) jet alone, while on the right-hand side a symmetric cut on the two hardest jets has been used.

exhibited a $16 \%$ excess which dropped to $1 \%$ when omitting the $37.5<p_{\mathrm{T}}<40.0 \mathrm{GeV}$ bin in the underlying Born $p_{\mathrm{T}}$ distribution, while using the $k_{\mathrm{T}}$ algorithm we find the excess is $16 \%$, reducing to less than $4 \%$ when the same bin is omitted from the $\left\langle p_{\mathrm{T}}\right\rangle$ distribution. Thus our conclusions based on analysing the underlying Born transverse momentum distribution can certainly be understood as being unequivocally physical.

Before leaving this discussion, we would like to point out that, in a recent publication [35], the problem of large, unphysical NLO corrections in dijet production has also been considered from a different perspective. It will be interesting to compare our findings with those of that work.

\subsubsection{Features of the hardest emission}

In dijet production, the radiation generated by POWHEG is generally softer than the other two jets. In order to investigate its radiation pattern, in the left plot of figure 14 we display the transverse momentum of the third highest $p_{T}$ jet at the LHC, in events where the leading jet has $p_{\mathrm{T}}$ greater than $40 \mathrm{GeV}$, while on the right we show the same spectrum, with the second jet also subject to the same cut. A slight kink is visible at around $20 \mathrm{GeV}$ in the former case and at $40 \mathrm{GeV}$ in the latter. In the first instance, this feature can be ascribed to the fact that the majority of selected events will be such that the hardest jet is near the cut, with the balancing second and third jets having a relatively small angular separation, thus bounding the $p_{\mathrm{T}}$ of the third jet to be less than $20 \mathrm{GeV}$. In the second case, the fact that the cross section falls rapidly as the transverse momentum of the two leading jets increases, favours them having $p_{\mathrm{T}}$ close to $40 \mathrm{GeV}$ in events passing the cut, hence, by definition, the $p_{\mathrm{T}}$ of the third jet will tend to be limited to this value. These arguments are easier to understand by considering the fixed-order predictions, since resummation effects in the POWHEG case add another layer of subtlety in the low $p_{\mathrm{T}}$ region. Lastly, we point out 

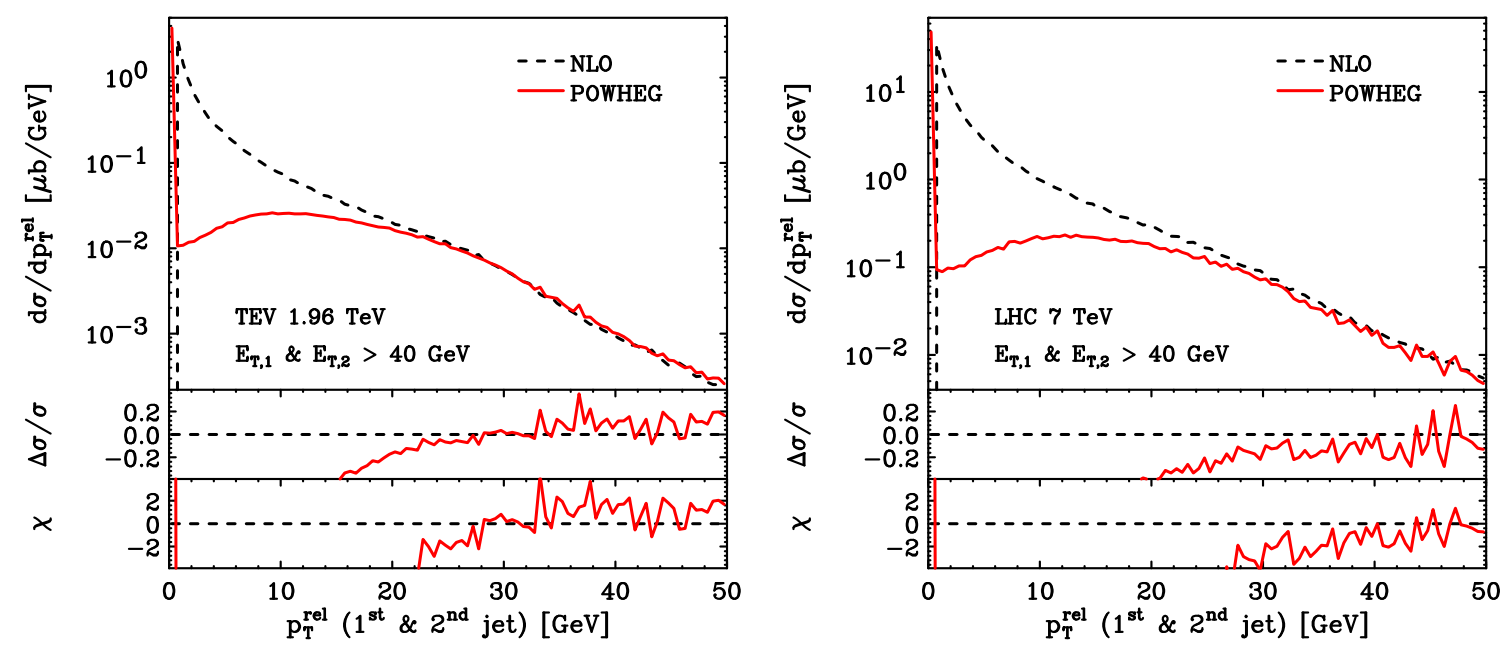

Figure 15. On the left- and right-hand side we show the distribution of the $p_{\mathrm{T}}^{\text {rel }}$ distribution for the two highest transverse-momentum jets, as given by POWHEG (solid red) and NLO QCD (black dashes), at the Tevatron and LHC.

that the vanishing of both the NLO and POWHEG distributions below $3 \mathrm{GeV}$ arises from our use of the D0 jet algorithm, which discards jets with transverse energy below $3 \mathrm{GeV}$.

With the origins of the kinks understood we can safely say that, for both distributions shown, the differences of the NLO predictions with respect to those of POWHEG display the usual features. In the low- $p_{\mathrm{T}}$ region we see that the NLO results tend to diverge while those of POWHEG are affected by Sudakov damping. At larger transverse momenta, the Sudakov suppression effects disappear and the POWHEG result tends to the fixed-order one, multiplied by a factor of $\bar{B}\left(\Phi_{B}\right) / B\left(\Phi_{B}\right)$ from the hardest emission cross section [7,8]. Note that, from the point of NLO accuracy, the presence or absence of such a factor is formally irrelevant, since, in the high- $p_{\mathrm{T}}$ regime, the distribution of events is governed by the real emission cross section, about which the $\bar{B}\left(\Phi_{B}\right) / B\left(\Phi_{B}\right)$ factor produces modifications of NNLO significance only. For a more detailed explanation of this point, in the context of Higgs boson production via gluon fusion, we refer the reader to refs. [36, 37].

\subsubsection{Jet structure: the $p_{\mathrm{T}}^{\text {rel }}$ distribution}

In order to investigate the jet structure, in figure 15 we show the scalar sum of the relative transverse momenta of the particles in the $j^{\text {th }}$ jet, $p_{\mathrm{T}}^{\text {rel, } j}(j=1,2)$, defined with respect to the jet axis, in the frame where the jet has zero rapidity:

$$
p_{\mathrm{T}}^{\mathrm{rel}, j}=\sum_{i \in j} \frac{\left|\vec{k}_{i} \times \vec{p}_{j}\right|}{\left|\vec{p}_{j}\right|},
$$

where $k_{i}$ denotes the momentum of the $i^{\text {th }}$ particle, and $p_{j}$ is the momentum of the $j^{\text {th }}$ jet. As throughout this section, we have compared the predictions of the POWHEG hardestemission cross section (solid red) against the corresponding fixed-order predictions (black dashes). Each of the two hardest jets in the event gives rise to an entry in this histogram, 
however, in dealing with the POWHEG hardest-emission events and their fixed-order counterparts, the final-states only consist of two or three partons. This being so, in the first bin we see an accumulation of events in the POWHEG case and a negative result in the fixedorder prediction due to two-parton counter events. All other bins are filled by three-parton events, which are clustered into two jets.

In both plots we see that the fixed-order result exhibits a mild (logarithmic) divergence for small values of $p_{\mathrm{T}}^{\text {rel }}$. On the other hand, the POWHEG prediction displays strong Sudakov damping and a sharp positive peak for $p_{\mathrm{T}}^{\text {rel }}=0$. Although the POWHEG predictions for particularly exclusive observables (like the one we are considering here) may appear better behaved than the pure NLO one, they are also plainly unphysical, as evidenced by the first bin of these histograms. We reiterate that, in terms of radiation, these bare POWHEG events contain only the hardest emission. Besides the erroneous peak, the Sudakov suppression here is also somewhat spurious, since it is not simply due to the inhibition of radiation around the leading jet axis but also to the fact that no harder radiation is allowed to come from the other initial-state or final-state partons. Only after the POWHEG output is interfaced to a parton shower can the shape of the Sudakov region be correctly modeled, with the peak at $p_{\mathrm{T}}^{\mathrm{rel}}=0$ also disappearing.

These $p_{\mathrm{T}}^{\text {rel }}$ distributions remind us the limitations of the POWHEG hardest-emission cross section alone: despite offering, in general, a greatly improved description with respect to fixed-order methods, it will naturally fail to describe exclusive observables sensitive to the emission of more than one parton.

\subsection{Parton showering and hadronization}

In this section we investigate the effects of showering and hadronization on the POWHEG results discussed previously. When showering the hardest emission event with PYTHIA 6.4.21 [38] and HERWIG $6.510[39,40]$ we have used their default settings, with no underlying event and multiple-parton interactions. Jet reconstruction was performed using jet algorithms and parameters specified at the beginning of section 3 .

We remind the reader that the shower programs should veto radiation harder than the $p_{\mathrm{T}}$ of the POWHEG generated hardest emission, which is passed to the shower program in the variable SCALUP, in the "Les Houches Interface for User Processes" common block [41, 42]. When POWHEG is interfaced to angular-ordered shower programs like HERWIG, in order to preserve coherent soft emissions from bunches of collinear partons, vetoed truncated showers should also be introduced [7]. These are not available at the moment in HERWIG. Previous studies [37, 43], carried out in the framework of the HERWIG++ collaboration [10], have shown that these effects are very modest. When POWHEG is interfaced to a virtualityordered shower, like the one in the old PYTHIA versions, coherent soft emission is absent anyhow, so there is no point in worrying about truncated showers. On the other hand, when interfacing POWHEG to transverse-momentum ordered dipole shower models, like the new PYTHIA shower, coherence is preserved by construction, and truncated showers are not required. 

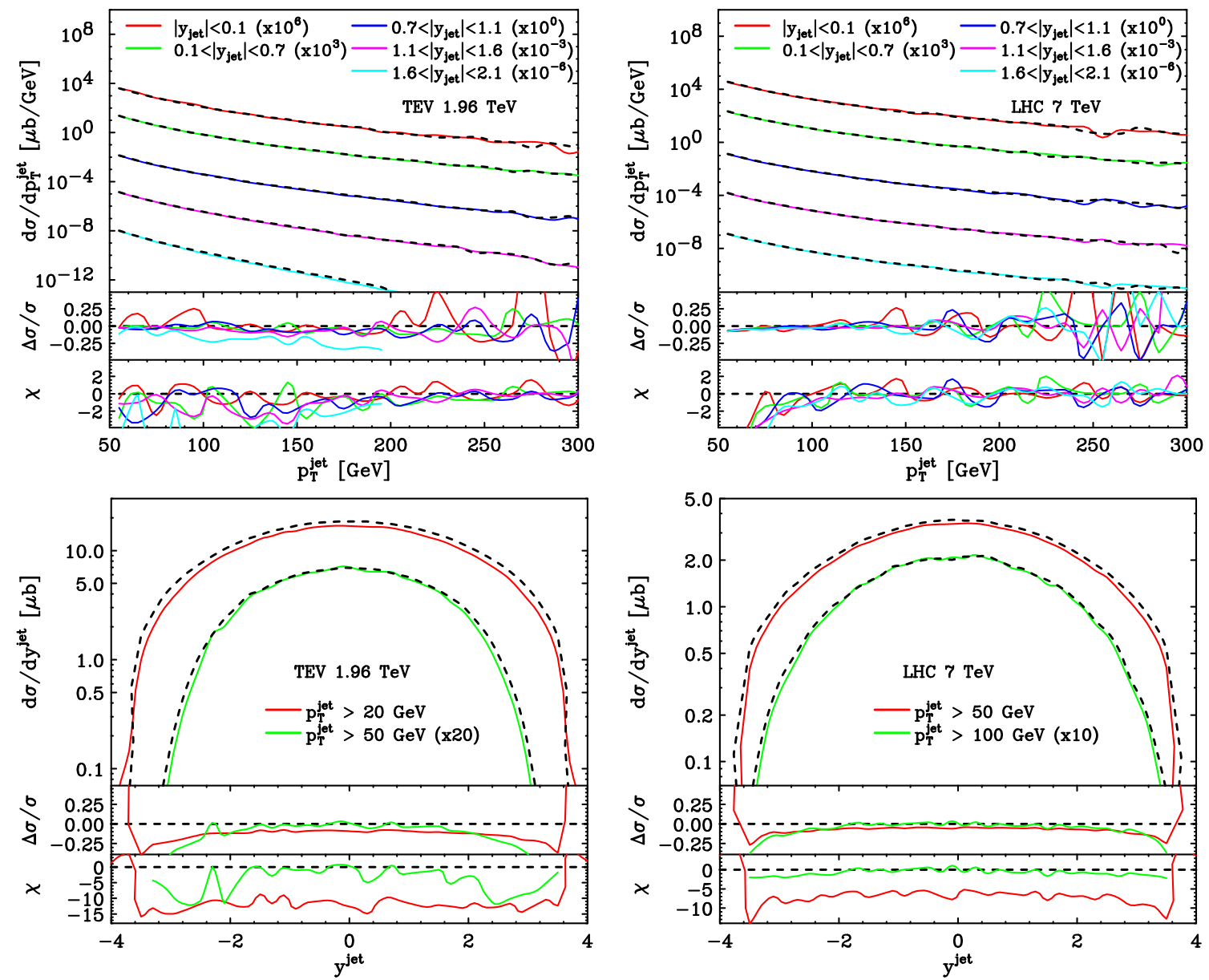

Figure 16. POWHEG hardest-emission cross sections for the inclusive jet transverse-momentum and rapidity spectra, i.e. prior to showering (black dashes), overlaid on those obtained when the corresponding hardest-emission events have been showered with PYTHIA (solid coloured lines). The transverse-momentum spectra have been binned according to the rapidities of the jets, the results being ordered from top to bottom with increasing jet rapidity, while in the case of the rapidity spectra their ordering is as in the legend.

\subsubsection{Inclusive distributions}

In figure 16 we show, again, the inclusive jet transverse-momentum and rapidity spectra, where this time the black dashed lines pertain to the POWHEG hardest-emission events, and their coloured counterparts correspond to those obtained from the analysis of the aforesaid events when evolved to the hadron level by PYTHIA. In general the two sets of results are seen to agree well, as expected given that these are very inclusive quantities. One can also see that the redistribution of the momenta of the hard partons, among those generated by the shower, slightly depletes the transverse momentum of the jets, leading to a slight excess of the parton level, hardest-emission cross section with respect to the hadron level results. 

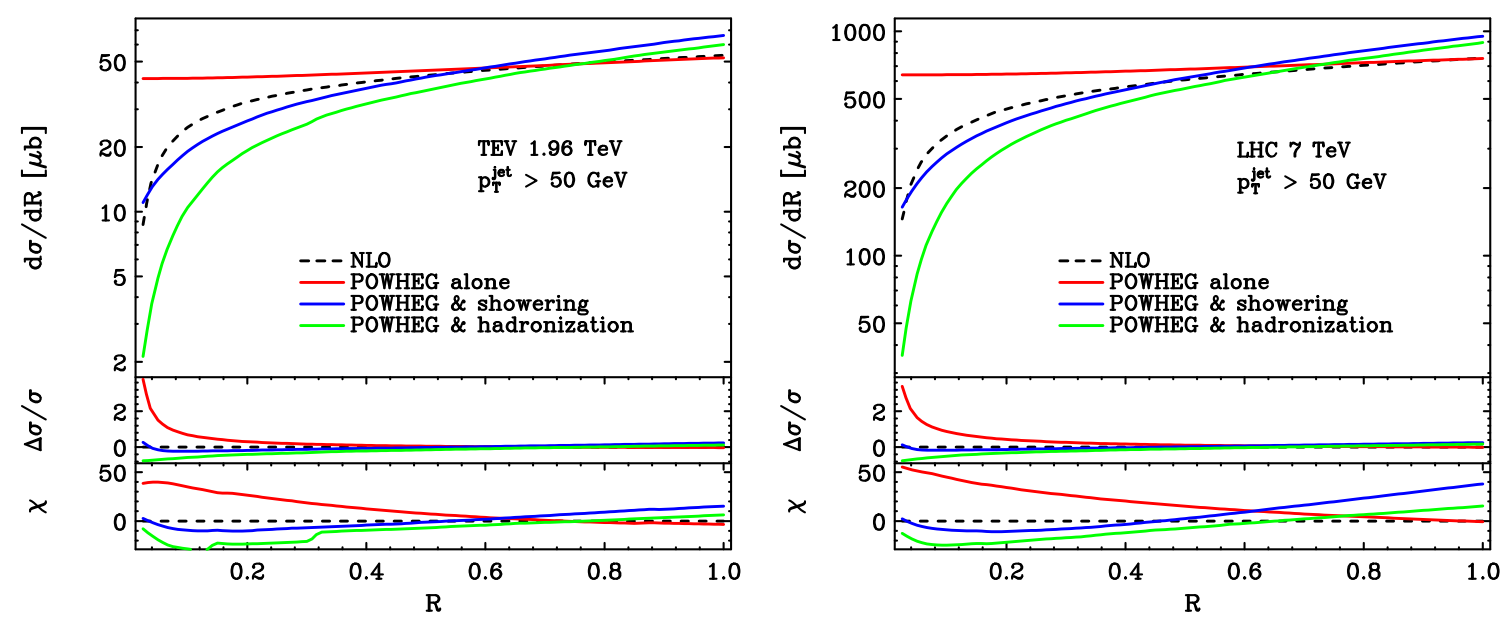

Figure 17. The inclusive jet cross section for jets with transverse momenta in excess of $50 \mathrm{GeV}$, as a function of the jet radius parameter $R$. The black dashed line represents the fixed-order NLO prediction, while the coloured lines correspond to those obtained with bare POWHEG events (red), showered POWHEG events (blue) and showered and hadronized POWHEG events (green).

\subsubsection{The $R$ dependence of the jet cross section}

As stated in the previous section, in our studies thus far, we have always taken the value of the jet radius parameter, $R$, to be 0.7 . This relatively large choice ensures good agreement between partonic and showered jets. It is time now, however, to assess the $R$ dependence of the jet cross section, especially in view of the fact that at the LHC smaller values are often used. In figure 17 we display the $R$ dependence of the inclusive jet cross section, for jets with transverse momenta greater than $50 \mathrm{GeV}$, at various levels of the simulation chain: NLO, hardest emission, showered, and hadron-level events. The same configuration of PYTHIA as above has been used for the last two steps. Unsurprisingly we can see that for $R=0.7$ and above there is generally good agreement between the different predictions, while the opposite is not true.

We notice, in particular, a marked difference between the pure NLO and bare POWHEG results, which deserves some explanation. First of all, we remind the reader that the $R$ dependence, as an observable, is similar to the transverse momentum of the third jet, or to the $p_{\mathrm{T}}^{\mathrm{rel}}$, in that it is not influenced by virtual corrections, but only by the real radiation. We thus expect it to display, at the NLO level, an unphysical behaviour, in the form of a logarithmic divergence at small $R$, as observed. We also expect that the (unshowered) POWHEG result will smear this divergence with a Sudakov form factor. This is indeed seen to be the case but the effect of the smearing is so strong that the POWHEG results displays a very mild $R$ dependence, up to the point where the NLO and the POWHEG predictions merge. On the other hand, the showered results display a stronger (and more physical) $R$ dependence at small $R$.

Although strange, it is easy to convince ourselves that this behaviour is correct. In fact, as in the case of the $p_{\mathrm{T}}^{\text {rel }}$ distribution, since the bare POWHEG events comprise of a single emission, the $R$ dependence induced by further emissions from the initial-state and final-state recoiling partons is absent. The fact that only one of the four partons in 

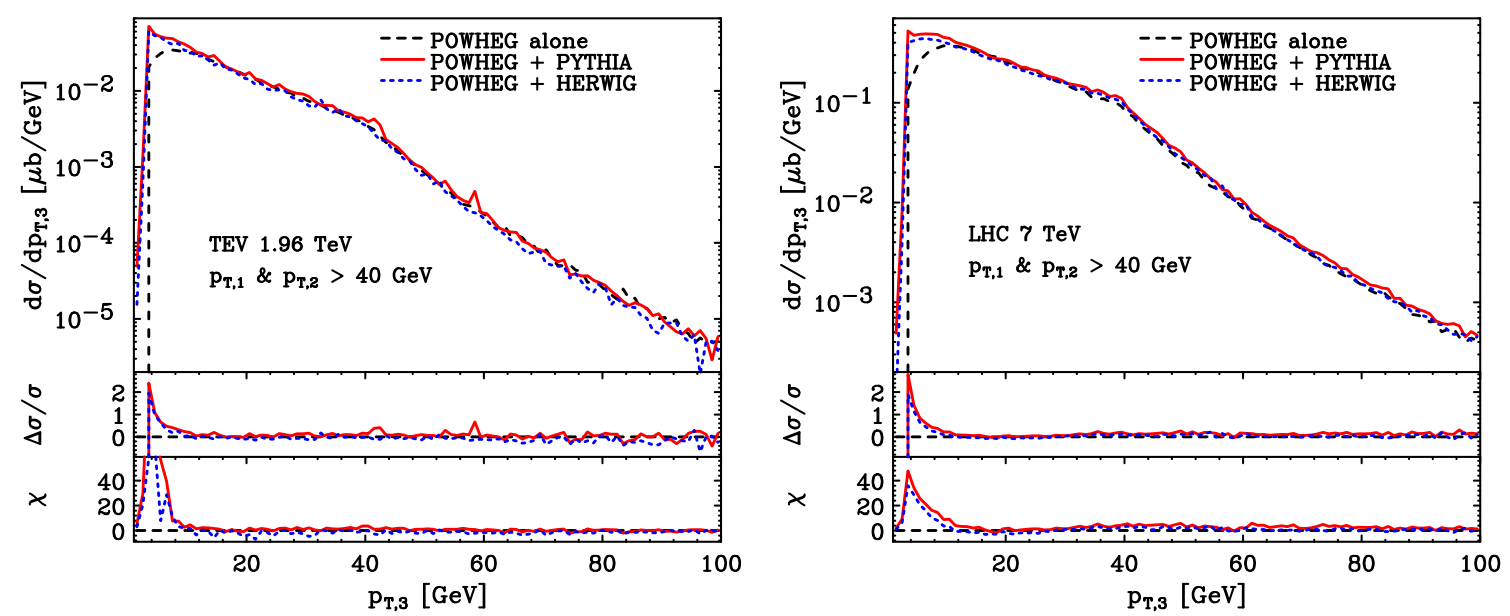

Figure 18. The transverse-momentum spectrum of the third hardest jet in dijet production. The dashed black lines correspond to the predictions of the POWHEG hardest-emission cross section, while the solid red and dotted blue lines correspond to showering the associated events using PYTHIA and HERWIG, omitting underlying-event activity.

the underlying Born configuration emits suggests that roughly $3 / 4$ of the cross section should exhibit no $R$ dependence. One should also consider that the POWHEG Sudakov form factor will inhibit small angle radiation, thus making the $R$ dependence even smaller. Furthermore, in the hardest-emission events, the Sudakov suppression is much stronger than the one that applies to a single radiating parton, since it also includes contributions corresponding to the non-emission of the other partons. Thus, the bare POWHEG prediction of the $R$ dependence is not realistic; the correct behaviour is only obtained here after the subsequent shower is turned on.

This is fully analogous to the case of the $p_{\mathrm{T}}^{\text {rel }}$ distribution in figure 15, where the POWHEG distribution displays a (delta function) peak at $p_{\mathrm{T}}^{\mathrm{rel}}=0$ and a broad Sudakov shoulder. Further showering naturally transforms this peak into other Sudakov shoulders, one for each jet in the event, overlapping with the original one and making it narrower. In the present case, the subsequent shower turns the flat $R$ dependence due to the non-emitting partons into a positive slope, since more energy is dissipated outside the jet cone, as $R$ decreases.

We notice that hadronization effects tend to further increase the slope of the $R$ dependence. This is a known effect, since hadron formation will further randomize the particles' momenta, driving even more energy out of the cone. It is also known that the underlying event (not included here) counteracts the effect of hadronization, since it generates soft hadrons, that bring more energy into the jet cone, with a probability proportional to its area.

\subsubsection{More exclusive distributions}

We now consider the effects of showering and hadronization on more exclusive observables. We use again PYTHIA with the aforementioned default setting, and HERWIG [39, 40] with default values of the parameters. In both cases, no multi-particle and underlying-event effects are considered. 

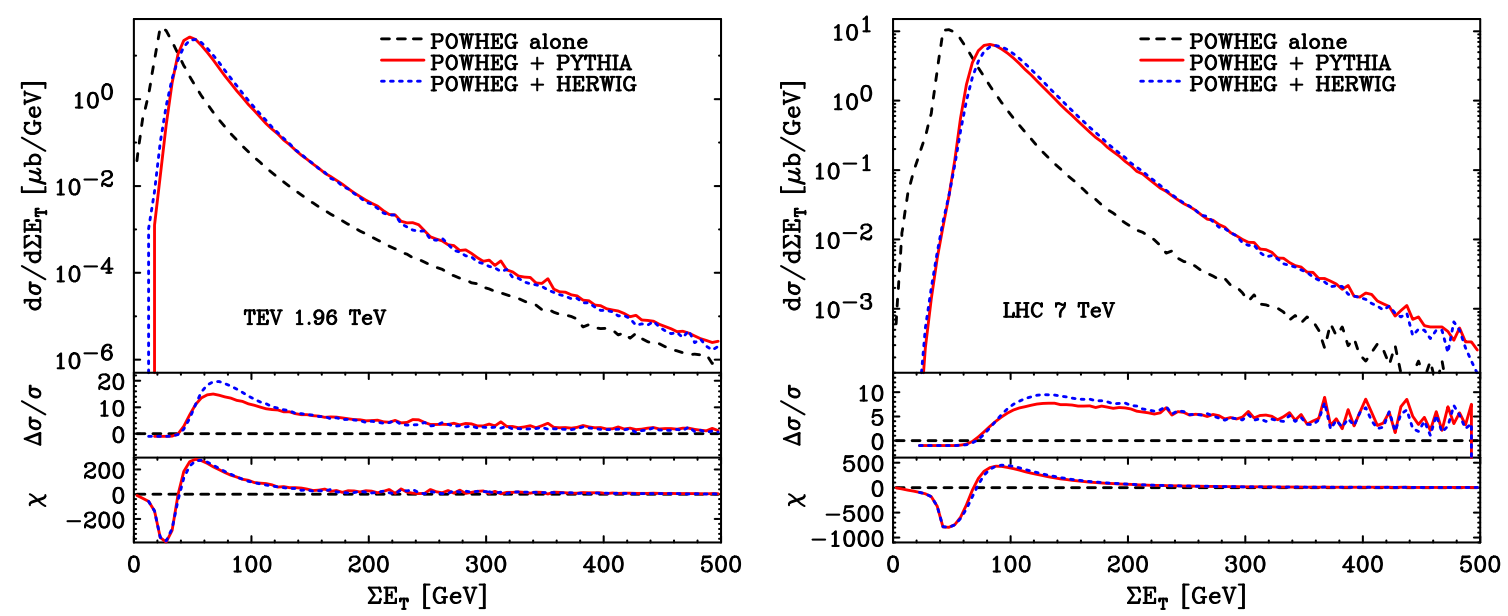

Figure 19. The total transverse-energy spectrum, namely, the scalar sum of the transverse energies of all particles in each event, at Tevatron (left) and LHC (right) energies. As in figure 18, the black dashed lines represent the predictions of the POWHEG hardest-emission cross section alone, while the solid red and dotted blue lines correspond to showering the hardest emission events using PYTHIA and HERWIG, neglecting underlying-event effects.

In figure 18 , we re-examine the $p_{\mathrm{T}}$ spectrum of the third jet. Here we see that the action of showering and hadronization is especially manifest for low $p_{\mathrm{T}, 3}$ values. The behaviour shown here supports the analysis of the $R$ dependence: the effect of further showering undoes the strong Sudakov suppression of the low $p_{\mathrm{T}, 3}$ region, imposed by the POWHEG hardest-emission cross section, yielding a result that is more peaked there. In comparing to the right-hand plot in figure 14 we can see, however, that the increase due to further showering is still much below that of the NLO result. In other words, some Sudakov suppression correctly remains, although not as much as the bare POWHEG result displays. Lastly we remark that, in contrast to the bare POWHEG predictions, the showered events populate the region of $p_{\mathrm{T}}$ below $3 \mathrm{GeV}$, since the cut in the D0 jet algorithm applies to the transverse energy of the jet cones, which can be smaller than the $p_{\mathrm{T}}$ for the showered events.

The effect of parton showering and hadronization on the scalar sum of the transverse energy of all particles is shown in figure 19. In the results obtained with both PYTHIA and HERWIG we find a dramatic shift in the distribution to higher energies, of approximately $50 \mathrm{GeV}$. This result should, however, not be considered alarming. The activity accompanying the showered events always leads to large multiplicities and, for example, even the production of 100 soft particles, with momenta of the order of $500 \mathrm{MeV}$, is capable of raising the total transverse energy by $50 \mathrm{GeV}$. We should thus consider this large discrepancy between the unshowered and the showered result as fully understandable.

\section{Phenomenology}

In this section, we compare the predictions for dijet production obtained with the POWHEG BOX, fully showered by PYTHIA 6.4.21 [38], including hadronization, underlying-event and multi-particle effects as defined by the Perugia 0 tune, with some Tevatron and LHC pub- 
lished measurements. For ease of notation, in the following, we will refer to the theoretical results simply as the 'POWHEG results'. All the aforementioned effects from PYTHIA are always included.

In order to efficiently populate the high $-p_{\mathrm{T}}$ regions of phase space, we have always used weighted-event samples in this section. On rare occasions we find that, when using PYTHIA for showering, large spikes with large errors appear in the distributions. We have not observed any such behaviour in the bare POWHEG output. We have concluded that this unpleasant feature is due to the fact that, in exceptional cases, low transverse-momentum hardestemission events, with a large weight, can be promoted to high transverse-momentum events by showering. In order to circumvent the problem, on the few occasions on which it has occurred here, we have merged histograms obtained by showering the same hardest-emission events using PYTHIA runs with different random seeds, replacing these anomalous bins with spikes by the corresponding result obtained in other PYTHIA runs, with smaller error. Unfortunately, the only way guaranteed to avoid this problem is to use unweighted samples with different generation cuts to cover the whole transverse-momentum spectrum.

The purpose of this section is, to some extent, to validate our code with real data rather than performing exhaustive tests on jet physics. We thus limit ourselves to a single PDF set, CTEQ6M, and we use, as renormalization and factorization scale in the evaluation of $\bar{B}\left(\Phi_{B}\right)$, the transverse momentum of the underlying Born configuration, as described in section 2.2 .

\subsection{Multiple-parton interactions in PYTHIA and the POWHEG jet generator}

The recent versions of PYTHIA include a model of multiple-parton interactions (MPI) [44] that improves the description of the underlying event accompanying the hard scattering process. A multiple interaction is essentially a $2 \rightarrow 2$ parton scattering arising from the collision of the remnants of the incoming nucleons. In the PYTHIA model, the scale that limits the transverse momentum of these processes is set to be quite high, much above the scale of the hard process under examination. The logic behind this choice is well documented in the PYTHIA manual: when considering a process like, for example, $Z$ production, there is a finite probability that a pair of jets with transverse momenta larger than half the invariant mass of the $Z$ are produced, and the PYTHIA authors want these events to be effectively generated. However, if the process in question is jet production, this approach may lead to overcounting. Therefore, PYTHIA inhibits this behaviour when generating jets, limiting the MPI scale to that of the jets in the primary interaction. When interfacing PYTHIA to the POWHEG jet program (and, for that matter, to any matrix-element generator for jets) using the user-process interface, there is no way for PYTHIA to know that it is showering a jet process. The user should therefore force PYTHIA to limit the scale of the MPI to the hardness of the primary process. ${ }^{5}$ PYTHIA provides a method to do this, which is to set the parameter MSTP $(86)=1$. We have collected the sequence of PYTHIA calls we have used in appendix $\mathrm{A}$.

\footnotetext{
${ }^{5}$ In the dijet case this scale corresponds to the transverse momentum of the jets.
} 


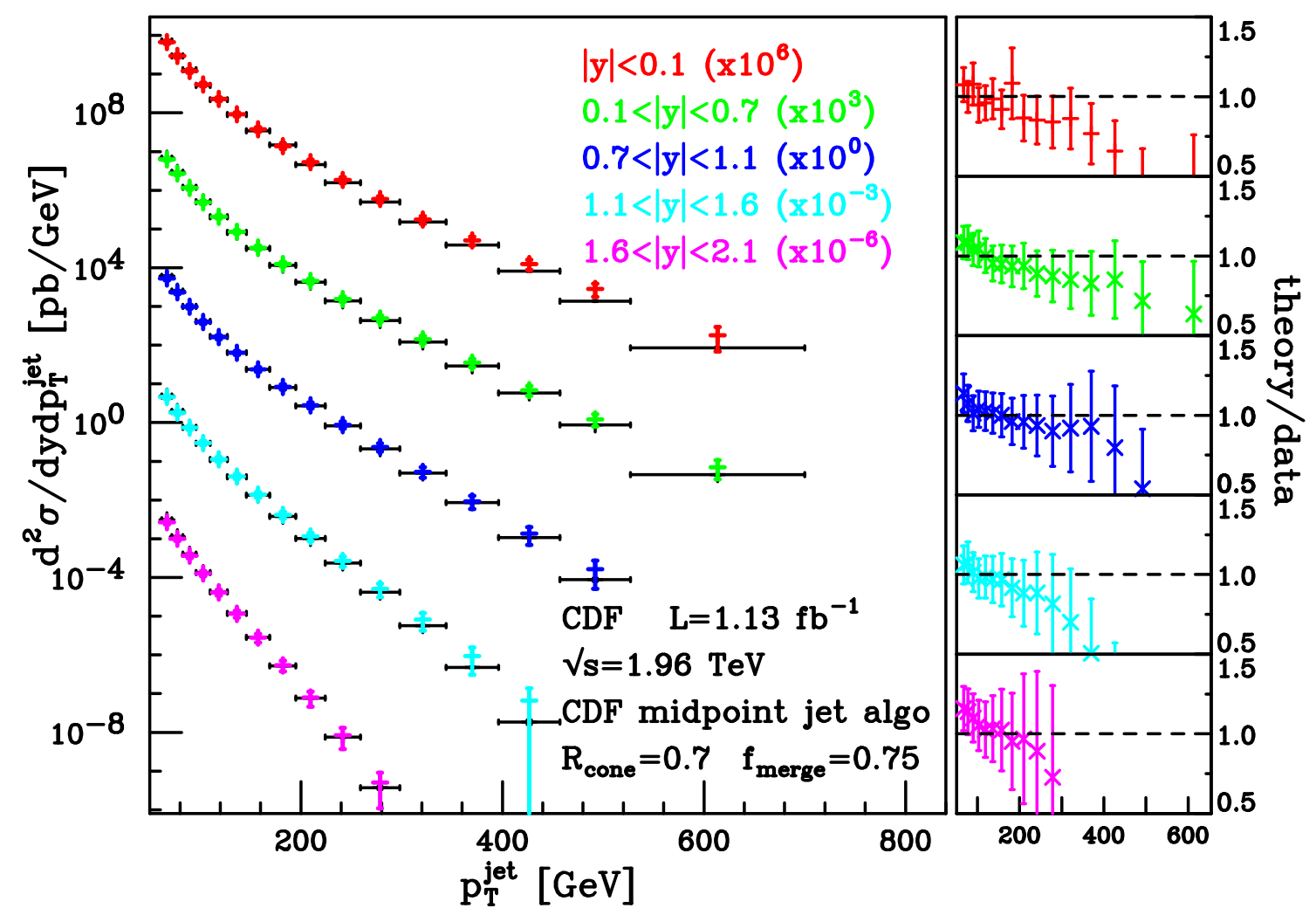

Figure 20. Predictions and experimental results for the double-differential inclusive jet cross section as a function of the transverse momentum of the jet $p_{\mathrm{T}}^{\text {jet }}$, for different bins of jet rapidity, $y$, as measured by the CDF Collaboration, using the cone-based midpoint jet algorithm. Black lines are the POWHEG+PYTHIA results (error bars are drawn too, even if almost invisible on the plot scale), while coloured bars are the experimental data (with errors represented as vertical bars) [31]. Data are shown from top to bottom in order of increasing rapidity.

\subsection{Tevatron results}

In this section we illustrate the comparison between the results obtained with the POWHEG BOX and data from the CDF and D0 Collaborations at the Tevatron, running at a centerof-mass energy of $1.96 \mathrm{TeV}$.

We have generated a sample of roughly 5 millions weighted events, with a cut on the Born transverse momentum of $1 \mathrm{GeV}$ and with $k_{\mathrm{T} \text {,supp }}=600 \mathrm{GeV}$, in order for the events to cover a region in transverse momentum up to $1 \mathrm{TeV}$. We have used the folding $1-5-1,{ }^{6}$ getting a fraction of negative-weight events below $5 \%$, that we have disregarded.

In figures 20 and 21 we plot the double-differential inclusive jet cross section as a function of the transverse momentum of the jet $p_{\mathrm{T}}^{\text {jet }}$, for different bins of jet rapidity $y$, as measured by the CDF Collaboration. Jets in figure 20 are reconstructed using the cone-based CDF midpoint jet algorithm [23], with a jet radius parameter $R=0.7$ and

\footnotetext{
${ }^{6}$ That is to say, setting ifoldcsi= 1 , ifoldy $=5$ and ifoldphi= 1 in the powheg.input file. See refs. $[22,30]$ for a detailed explanation.
} 


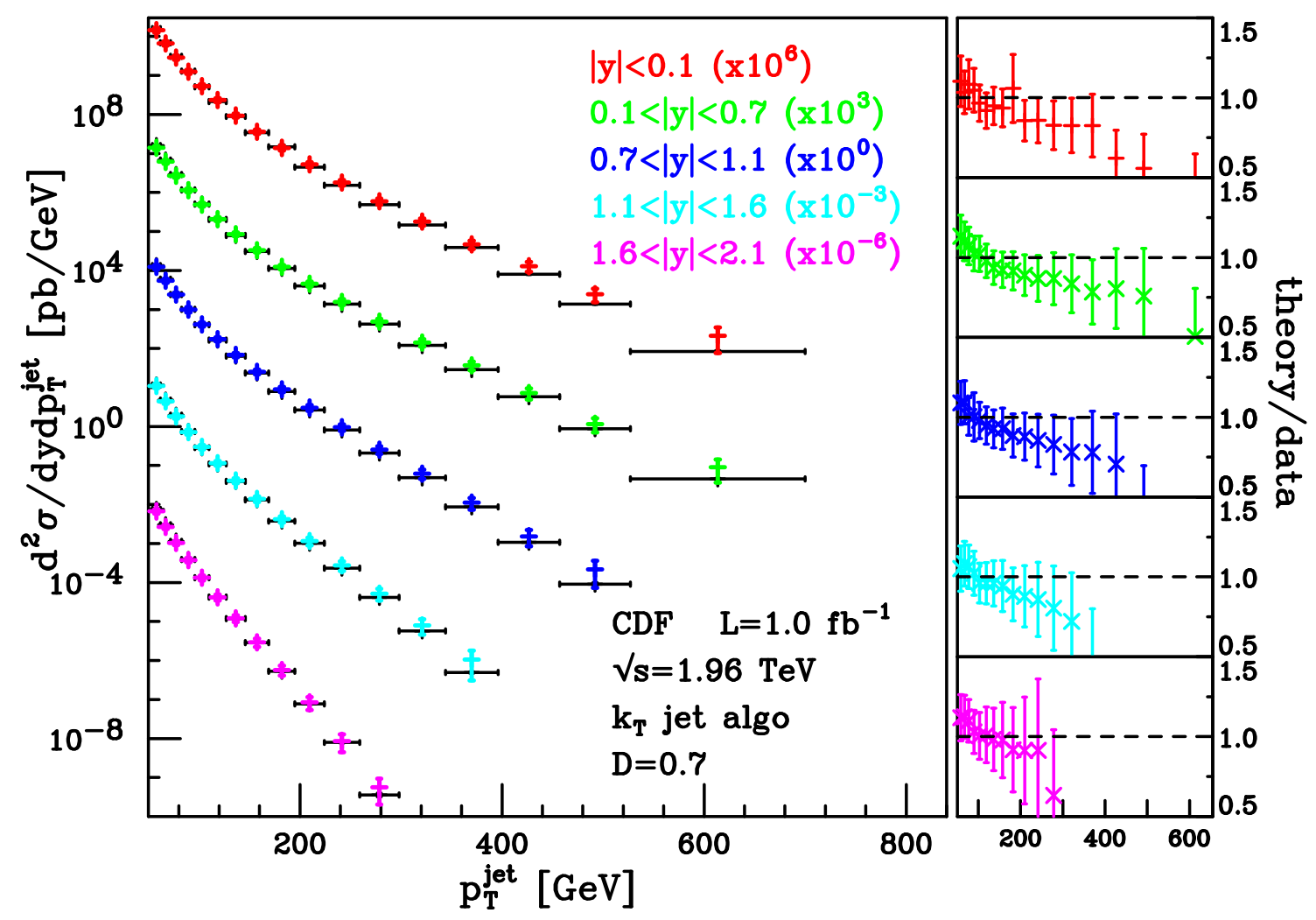

Figure 21. Predictions and experimental results for the double-differential inclusive jet cross section as a function of the transverse momentum of the jet $p_{\mathrm{T}}^{\text {jet }}$, for different bins of jet rapidity, $y$, as measured by the CDF Collaboration, using the $k_{\mathrm{T}}$ jet algorithm. Black lines are the POWHEG+PYTHIA results (error bars are drawn too, even if almost invisible on the plot scale), while coloured bars are the experimental data (with errors represented as vertical bars) [45]. Data are shown from top to bottom in order of increasing rapidity.

overlapping fraction $f=0.75$. After jet clustering, only jets with

$$
62 \mathrm{GeV}<p_{\mathrm{T}}^{\text {jet }}<700 \mathrm{GeV} \text { and }\left|y^{\text {jet }}\right|<2.1
$$

are kept. POWHEG results are shown as black lines, while the experimental data [31] are drawn as coloured bars, with errors obtained by summing in quadrature the statistical and the systematic errors.

In figure 21 we compare the POWHEG predictions with results from the analysis of ref. [45]. Jets are recombined using the $k_{\mathrm{T}}$ algorithm [28, 29], where the jet-size parameter $D$ has been set equal to 0.7 . Only jets with

$$
54 \mathrm{GeV}<p_{\mathrm{T}}^{\text {jet }}<700 \mathrm{GeV} \text { and }\left|y^{\text {jet }}\right|<2.1
$$

are kept.

We remark that, in refs. [31] and [45], data were compared to NLO calculations corrected for the parton-to-hadron correction factors. Here, the measured values are directly compared to the POWHEG results showered by PYTHIA, and the agreement is quite good, as can be seen from the ratios theory/data in figures 20 and 21 . 

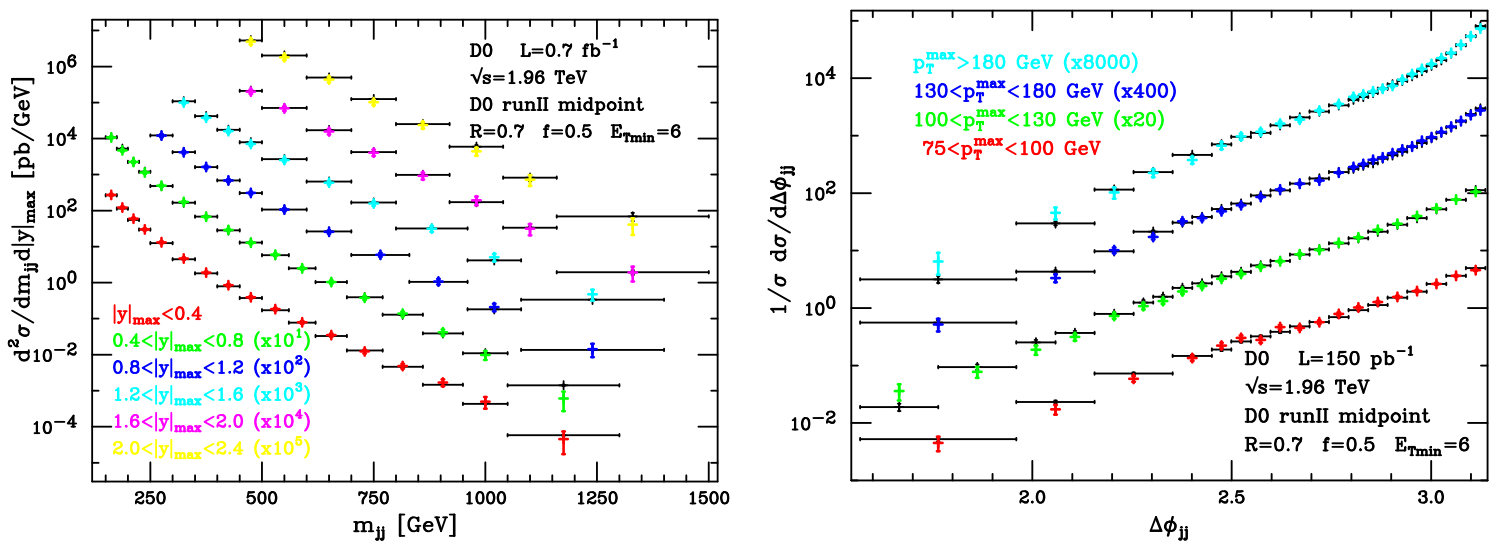

Figure 22. On the left, we confront the POWHEG+PYTHIA results with the D0 measurement [34] of the double-differential dijet cross section, as a function of the invariant mass of the two leading jets, $m_{j j}$, for different bins of $|y|_{\max }$, the maximum absolute value of the rapidity of the two jets. On the right-hand side, we show the dijet azimuthal decorrelation, $\Delta \phi_{j j}$, in bins of the transverse momentum of the leading jet $p_{\mathrm{T}}^{\max }[46]$. Black horizontal bars correspond to the POWHEG outputs. D0 data are shown in colour, with experimental errors given by the vertical bars. Data are shown from top to bottom in order of increasing rapidity on the left, and of decreasing $p_{\mathrm{T}}^{\max }$ on the right.

In figure 22, we have performed a comparison between the POWHEG results and D0 data [34] for the invariant mass of the two leading jets and for the azimuthal angle between them. For both plots, we have used the seed-based D0 run II midpoint cone algorithm, with $R=0.7$, overlapping fraction $f=0.5$ and with minimum jet $E_{\mathrm{T}}$ parameter equal to $6 \mathrm{GeV}$, as in ref. [34]. In the left plot, we display the double-differential jet cross section as a function of the invariant mass of the two leading jets, $m_{j j}$, for different bins of $|y|_{\max }$, the maximum absolute value of the rapidity of the two jets. A minimum cut on the transverse momentum of the two leading jets is imposed, i.e. $p_{\mathrm{T}}^{\text {jet }}>40 \mathrm{GeV}$. Data [34] are shown in colour with vertical error bars, while the POWHEG results are depicted in black. Good agreement is found between data and the POWHEG results over quite a wide range of values of the dijet mass and of the rapidity intervals.

In the right plot, we show the azimuthal separation of the two hardest jets, $\Delta \phi_{j j}$. We require the hardest and next-to-hardest jets to have $p_{\mathrm{T}}^{\text {jet }}>75 \mathrm{GeV}$ and $p_{\mathrm{T}}^{\text {jet }}>40 \mathrm{GeV}$ respectively. In addition, jets must have central rapidities, i.e. $\left|y_{\text {jet }}\right|<0.5$, as in the analysis of ref. [46]. Results are shown in bins of $p_{\mathrm{T}}^{\max }$, the transverse momentum of the leading jet. Notice that the results are normalized to unity, so that the prediction power of POWHEG is less evident in this plot. For this physical variable, the NLO results becomes negative as $\Delta \phi_{j j} \rightarrow \pi$, i.e. as the third parton become soft or collinear to one of the other twos. Instead, the POWHEG curves are finite, since the Sudakov form factor resums the leading-logarithmic divergences as this limit is approached. On the other hand, hard jets contribute in the region where $\Delta \phi_{j j}$ gets smaller, so that we do not expect a perfect agreement with the POWHEG curves, that at most produces 3 hard jets.

Colour coherence effects have been observed and studied at CDF, in ref. [47]. In that paper, variables sensitive to interference effects have been identified and measured. The 


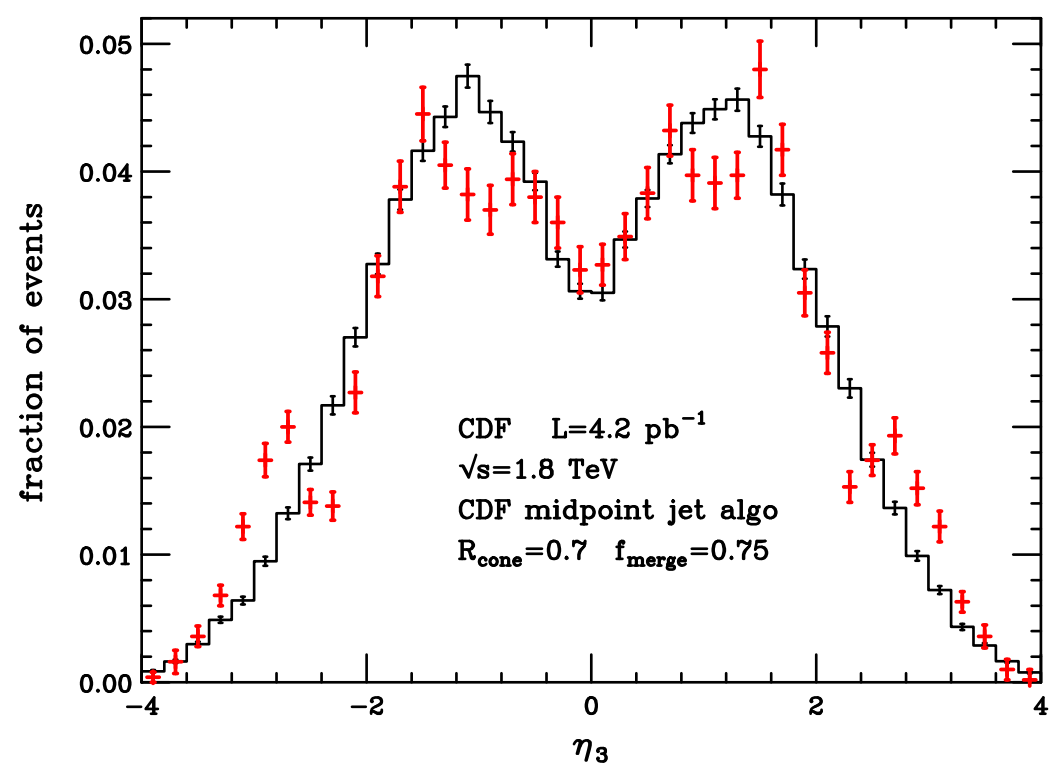

Figure 23. The pseudorapidity spectrum of the third hardest jet (ordered in the transverse energy) in three-jet events, requiring the two leading jets to satisfy $\left|\eta_{1}\right|,\left|\eta_{2}\right|<0.7,|| \phi_{1}-\phi_{2}|-\pi|<20^{\circ}$, with cuts on the transverse energies of the leading and third jet of $E_{\mathrm{T}, 1}>110 \mathrm{GeV}$ and $E_{T, 3}>10 \mathrm{GeV}$, as in ref. [47]. The red vertical bars are the collected data, while the POWHEG+PYTHIA result is shown as a black histogram.

data were collected in Run I at $1.8 \mathrm{TeV}$, on a sample of $4.2 \mathrm{pb}^{-1}$. We have generated a sample of about 5 million weighted events with the POWHEG BOX, having a Born minimum transverse momentum of $1 \mathrm{GeV}$ and a suppression factor $k_{\mathrm{T} \text {,supp }}=150 \mathrm{GeV}$. The folding used was 1-5-1, that gave rise to a fraction of $1 \%$ negative-weight events. We have applied the same jet algorithm and parameters used by other CDF analyses. Jets are then ordered with respect to the transverse energy, and not with respect to the transverse momentum. With this ordering, only events with at least three jets are selected. The two leading jets are required to be central in the detector, with pseudorapidities $\left|\eta_{1}\right|,\left|\eta_{2}\right|<0.7$, and to be back-to-back within $20^{\circ}$ in the transverse plane, ||$\phi_{1}-\phi_{2}|-\pi|<20^{\circ}$. The transverse energy of the first jet and of the third jet are required to be greater than $110 \mathrm{GeV}$ and $10 \mathrm{GeV}$. In figure 23 we plot the CDF results (red vertical bar) and the POWHEG prediction (black histogram) for the pseudorapidity of the third jet, $\eta_{3}$. The distribution is normalized to unit area. ${ }^{7}$ The colour-coherence feature is manifest as a central dip, not present if interference effects are not properly accounted for.

\subsection{LHC results}

The ATLAS Collaboration has recently published the first LHC data on dijet production at $7 \mathrm{TeV}$ [1]. To make a comparison with these data, we have generated a sample of about 5 million weighted events, with a cut on the Born transverse momentum of $1 \mathrm{GeV}$

\footnotetext{
${ }^{7}$ Note that the local distortions of the shape, such those at $\left|\eta_{3}\right|=2.5$, are due to uninstrumented regions of the detector, as pointed out in ref. [47].
} 


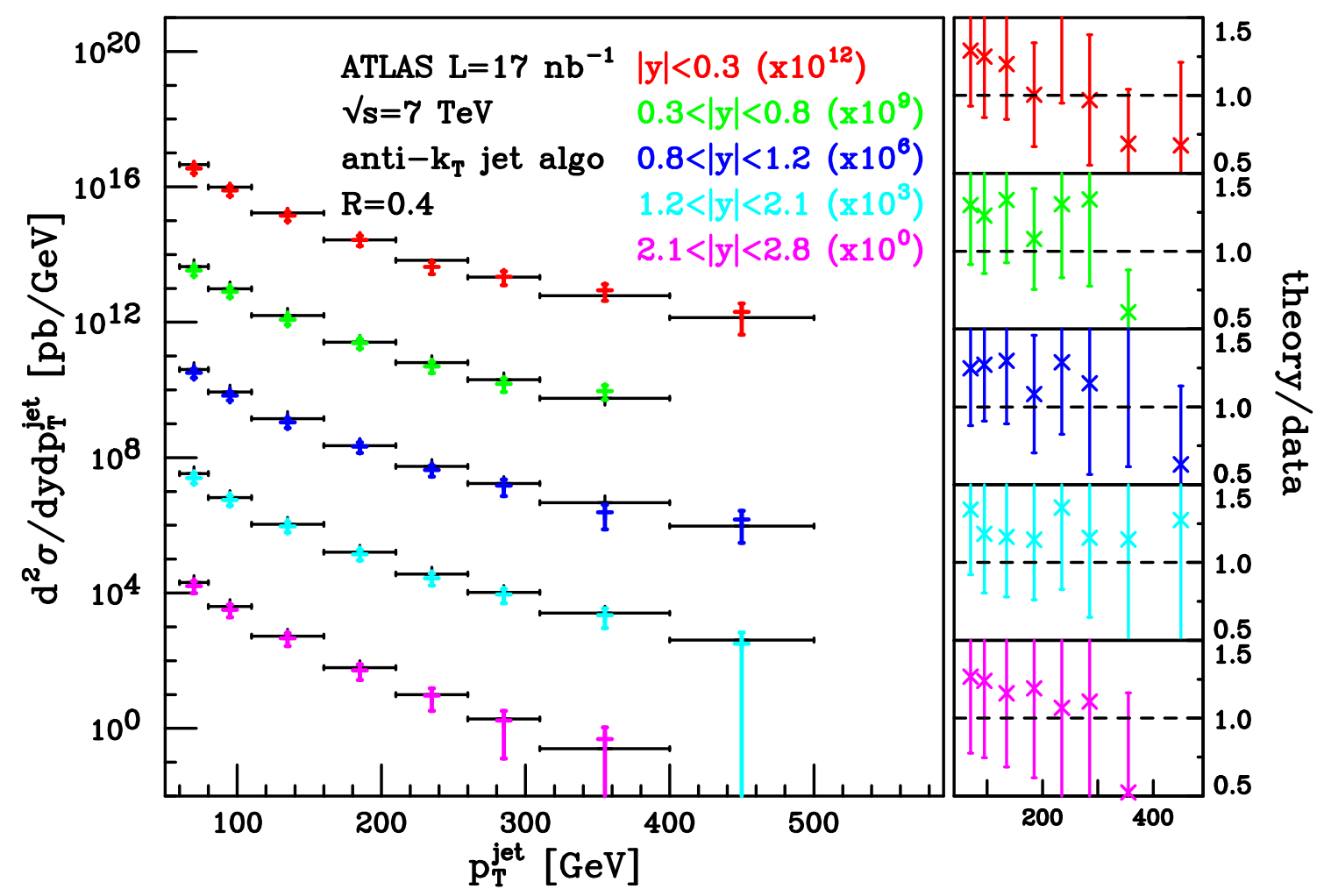

Figure 24. Predictions and experimental results for the double-differential inclusive jet cross section, as a function of the jet transverse momentum, $p_{\mathrm{T}}^{\mathrm{jet}}$, in bins of jet rapidity $y$ for the jets that pass the cuts of eq. (4.3). Black horizontal lines are the POWHEG+PYTHIA theoretical results (with errors, almost invisible at the scale of the plot). Coloured vertical bars describe the experimental data from ATLAS (systematic and statistical errors added in quadrature) [1]. Jets recombined using the anti- $k_{\mathrm{T}}$ algorithm with $R=0.4$. Data are shown from top to bottom in order of increasing rapidity.

and with $k_{\mathrm{T} \text {,supp }}=200 \mathrm{GeV}$, in order for the events to cover a region in the transverse momentum up to $600 \mathrm{GeV}$. We have used the folding 2-10-1, getting a fraction of negativeweight events equal to $1.6 \%$, that we have disregarded. Jets were reconstructed using the anti- $k_{\mathrm{T}}$ algorithm [48], which is infrared-safe at all orders. Furthermore, it has a simple geometrical interpretation in terms of cone-like jets. We adopted the same choice of resolution parameters used in ref. [1], i.e. $R=0.4$ and $R=0.6$.

In figures 24 and 25 we show the inclusive double-differential cross section, as a function of the jet transverse momentum, $p_{\mathrm{T}}^{\mathrm{jet}}$, in bins of jet rapidity $y$. In the first figure, results for $R=0.4$ are shown, while in the second one the value $R=0.6$ has been used. Only jets with

$$
p_{\mathrm{T}}^{\text {jet }}>60 \mathrm{GeV}, \quad\left|y^{\text {jet }}\right|<2.8
$$

are included in the plot. POWHEG results are shown as black horizontal lines, with width equal to the bin size used by ATLAS, and coloured vertical bars correspond to the experimental data of ref. [1], obtained by summing in quadrature the statistical and systematic errors. An additional overall uncertainty of $11 \%$, due to the measurement of the integrated 


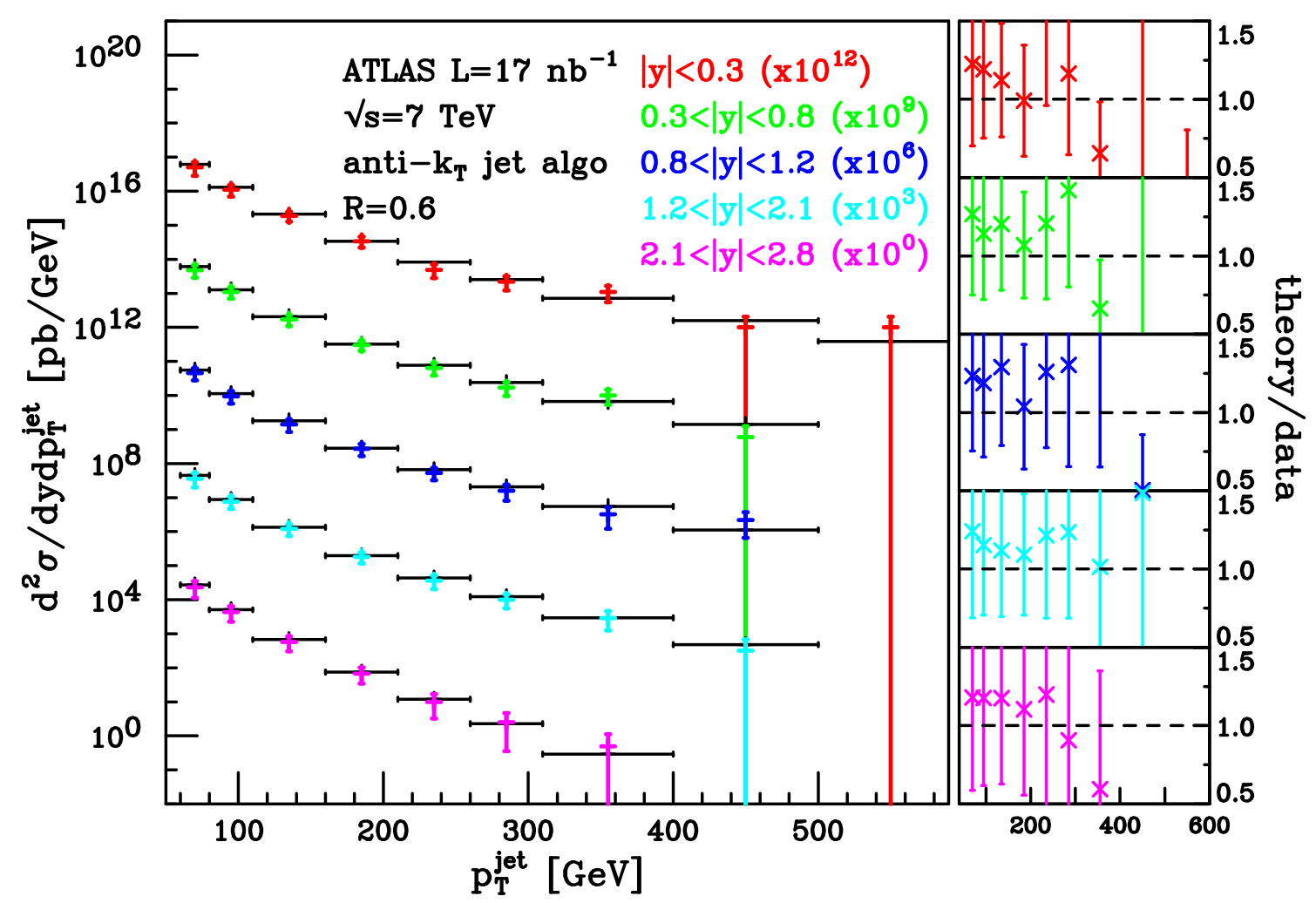

Figure 25. Predictions and experimental results for the double-differential inclusive jet cross section, as a function of the jet transverse momentum, $p_{\mathrm{T}}^{\mathrm{jet}}$, in bins of jet rapidity $y$ for the jets that pass the cuts of eq. (4.3). Black horizontal lines are the POWHEG+PYTHIA theoretical results (with errors, almost invisible at the scale of the plot). Coloured vertical bars describe the experimental data from ATLAS (systematic and statistical errors added in quadrature) [1]. Jets recombined using the anti- $k_{\mathrm{T}}$ algorithm with $R=0.6$. Data are shown from top to bottom in order of increasing rapidity.

luminosity, is also included in the error bars. The good agreement between theory and data is illustrated in the ratio plots on the right hand side of the two figures. As discussed in section 3.3.2, one expects that, as the jet radius $R$ increases, more particles are clustered in the jets that pass the cuts of eq. (4.3), and the agreement with theory is improved.

In figure 26, we compare the dijet double-differential cross section, as a function of the dijet mass $m_{j j}$ of the two leading jets, for different bins in $|y|_{\max }$, the maximum absolute rapidity of the two jets. The two leading jets are required to have

$$
p_{\mathrm{T}, 1}^{\mathrm{jet}}>60 \mathrm{GeV}, \quad p_{\mathrm{T}, 2}^{\mathrm{jet}}>30 \mathrm{GeV}, \quad\left|y_{1}^{\mathrm{jet}}\right|,\left|y_{2}^{\text {jet }}\right|<2.8
$$

In the left plot, results for the anti- $k_{\mathrm{T}}$ algorithm with $R=0.4$ are shown, while on the right the value $R=0.6$ has been used. Good agreement is found over the entire dijet mass and rapidity ranges.

In figure 27, we plot the dijet double-differential cross section as a function of the dijet angular variable $\chi$, for different ranges of the dijet mass $m_{j j}$ of the two leading jets. The 

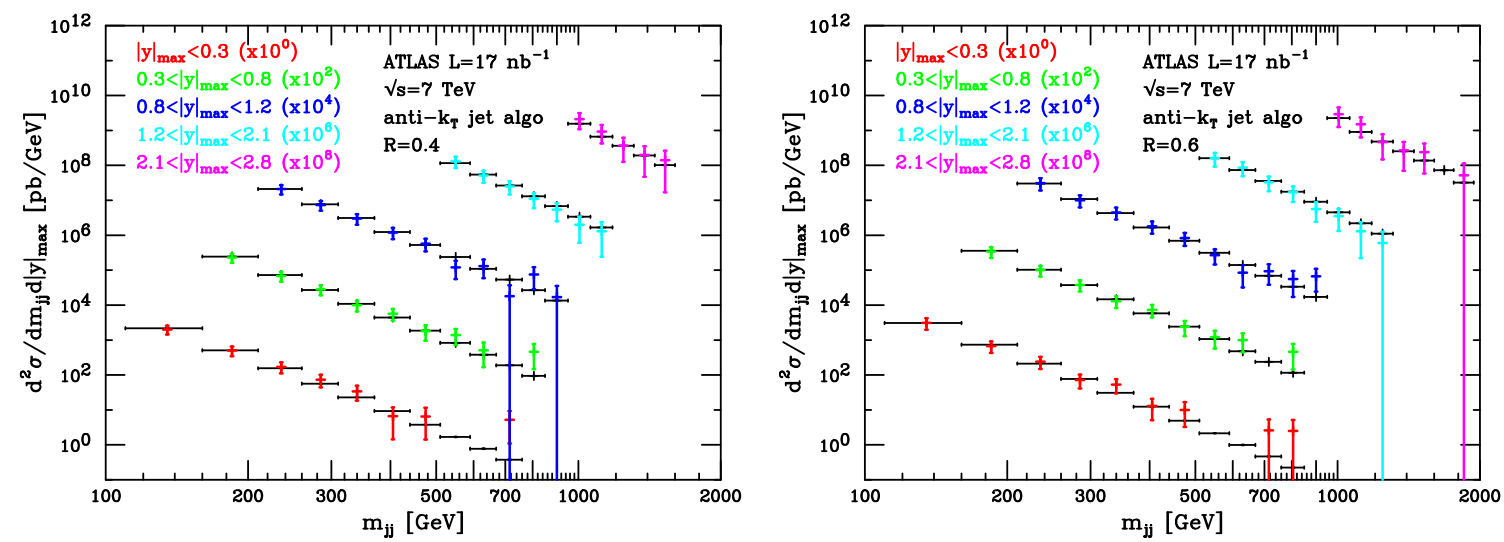

Figure 26. Predictions and experimental results for the double-differential inclusive jet cross section as a function of the dijet mass $m_{j j}$ of the two leading jet pair, for different bins in $|y|_{\max }$, the maximum absolute rapidity of the two jets. The cuts of eq. (4.4) are imposed on the two leading jets. Black horizontal lines are the POWHEG+PYTHIA theoretical results (with errors). Coloured vertical bars describe the experimental data from ATLAS (systematic and statistical errors added in quadrature) [1]. Jets are recombined using the anti- $k_{\mathrm{T}}$ algorithm with $R=0.4$ in the left plot, and $R=0.6$ in the right one. Data are shown from top to bottom in order of decreasing rapidity.
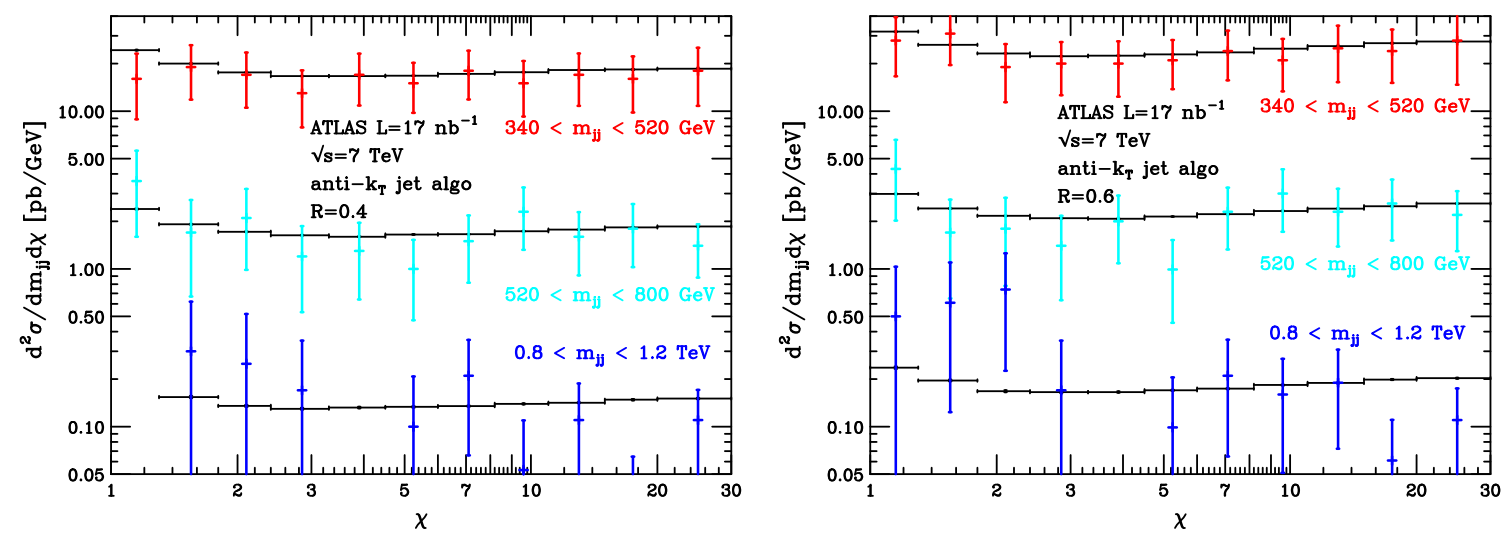

Figure 27. Predictions and experimental results for the dijet double-differential cross section as a function of the dijet angular variable $\chi$ (see eq. (4.5)), for different ranges of the dijet mass $m_{j j}$ of the two leading jets. Jets must satisfy the cuts of eqs. (4.4) and (4.6). Black horizontal lines are the POWHEG+PYTHIA theoretical results (with errors). Coloured vertical bars describe the experimental data from ATLAS (systematic and statistical errors added in quadrature) [1]. Jets recombined using the anti- $k_{\mathrm{T}}$ algorithm with $R=0.4$ in the left plot, and $R=0.6$ on the right.

$\chi$ angular variable is defined in terms of the rapidities of the two leading jets as

$$
\chi=\exp \left(\left|y_{1}-y_{2}\right|\right)
$$

Jets must pass the cuts of eq. (4.4) and, in order to reject events in which both jets are boosted in the forward or backward directions, they must satisfy

$$
\left|y_{\text {boost }}\right|<1.1
$$


where $y_{\text {boost }}=\left(y_{1}+y_{2}\right) / 2$. In the left-hand plot, we show results using the anti- $k_{\mathrm{T}}$ algorithm with $R=0.4$, while on the right-hand side we show the ones for $R=0.6$. These data are affected by quite large errors. The POWHEG results are in very good accord with data.

\section{Conclusions}

In this article we have presented a next-to-leading order parton shower simulation of dijet hadroproduction, based on the POWHEG formalism. We have assembled our generator with the aid of the POWHEG BOX toolkit, thereby reducing the task to that of writing a set of computer subroutines returning the Born, virtual, real and colour-correlated Born cross sections, for given input momenta and flavour structure, and to provide the Born-level phase space. By contrast, the validation and phenomenological studies following the construction of the simulation have proven subtle, with the generator output ultimately providing us with a better understanding of the dijet production process.

In previous studies with next-to-leading order calculations interfaced to parton showers (Nlops) it was found that the Nlops predictions for inclusive observables are in accord with those of conventional fixed-order computations. Marked differences between the fixedorder and NLOPS predictions have been essentially confined to exclusive quantities, sensitive to the emission of soft radiation. Conversely, in performing the same class of comparisons for inclusive quantities in dijet production, we have found conflicts. Only for the most inclusive observables, namely, the inclusive jet rapidity and transverse-momentum distributions, have we found the fixed-order and POWHEG results in perfect agreement. Other observables, such as the total cross section subject to a single cut on the leading jet transverse energy and, to a larger extent, the total cross section for the production of two jets with transverse energy above a common threshold, exhibit clear discrepancies, even for large jet radii.

We have studied these discrepancies in some detail and confirmed that they are symptomatic of a problem in the fixed-order computation. In regard to the cross sections with cuts on the two hardest jets, it was already shown in ref. [14] that the NLO real emission cross section develops large threshold terms, of the form $\Delta \log \Delta, \Delta$ being the difference of the transverse-energy cuts on the two leading jets. This large NLO correction thus spoils the convergence of the perturbative expansion and the corresponding NLO results display unphysical features. We have succeeded in reaching an analogous conclusion also in the case where a single cut is applied to the leading- $E_{\mathrm{T}}$ jet. We have used our program to assess how events with a common underlying Born kinematics reside or migrate on either side of the $E_{\mathrm{T}}$ cut boundary, exposing in both cases the logarithmic sensitivity of the cross sections to soft radiation.

As noted earlier, the problems of the NLO predictions in the presence of symmetric $E_{\mathrm{T}}$ cuts have been studied in the past and ways to resum these large corrections to all orders in perturbation theory have also been proposed [33]. In practice, the general consensus has been to warn against the use of symmetric cuts in experimental and theoretical dijet studies. Having said that, let us reiterate that, even in what one might regard as the maximally asymmetric case, in which the cut on the second jet is set to zero, the NLO 
prediction is logarithmically sensitive to soft radiation at the cut, albeit at the level of ten rather than one hundred percent corrections to the total cross section.

Since Sudakov logarithms are always resummed in the POWHEG approach, one can expect improved agreement with data for general observables, even the badly behaved inclusive observables mentioned above. Furthermore, insidious cases, when these large corrections may arise without being easily identifiable, are also handled correctly by the POWHEG generator. For example, in analysing the dijet invariant mass distribution we have found that a small, symmetric, transverse-momentum cut on the jets, ineffective for central jets, becomes instead effective for the highest rapidity bins, introducing a large mismatch between the NLO and POWHEG results in that region.

The reader may wonder what in particular is special about dijet production that gives rise to the problems that we have discussed. In NLOPS implementations concerning the production of massive objects, the distributions of the kinematic variables of the massive particle do not display any pathological behaviour. It is interesting, however, to try to look for the effects found in dijet production also in these cases, by building appropriate observables. In the case of $p p \rightarrow Z / \gamma+X \rightarrow l^{+} l^{-}+X$, for example, we have found that, in the presence of symmetric cuts of $45 \mathrm{GeV}$ on the transverse momentum of the leptons, the corresponding NLO computation exhibits essentially the same ill effects discussed here in the context of jet production. Thus, there is nothing special about dijet production in regards to this pathological behavior. NLO predictions for inclusive quantities in other processes can also exhibit similar weaknesses.

Finally, we have compared the results of our program to a wide variety of Tevatron and early LHC data. Although in these studies we have not included a full assessment of the theoretical uncertainties, we have seen that our predictions are in very pleasing agreement with the experimental measurements. We believe that a more thorough study using our program can only be performed in the framework of experimental collaborations studying jet physics. In particular, Monte Carlo tuning of hadronization and underlying event parameters should probably be performed again using the POWHEG dijet program, in view of the sensitivity of jet measurements to these features.

The code of our generator can be accessed in the POWHEG BOX svn repository: svn://powhegbox.mib.infn.it/trunk/POWHEG-BOX,

with username anonymous and password anonymous.

\section{Acknowledgments}

We wish to thank T. Sjöstrand for very useful exchanges and, in particular, for clarifying the use of the PYTHIA simulation of multiple interactions in jet processes. We also wish to thank E. Feng, P. Francavilla, C. Roda and G. Salam for useful correspondence. 


\section{A PYTHIA and HERWIG settings}

The sequence of PYTHIA calls we have used in the calculation of the results presented in section 3 is the following:

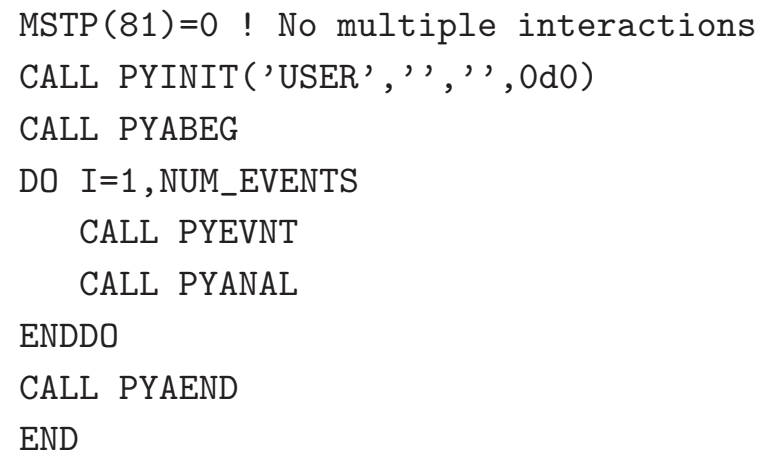

HERWIG was run setting the following parameters after the call to HWIGIN:

PTRMS $=2.5 \mathrm{D} 0$

$\mathrm{PRSOF}=0$

Open Access. This article is distributed under the terms of the Creative Commons Attribution Noncommercial License which permits any noncommercial use, distribution, and reproduction in any medium, provided the original author(s) and source are credited.

\section{References}

[1] ATLAS collaboration, G. Aad et al., Measurement of inclusive jet and dijet cross sections in proton-proton collisions at $7 \mathrm{TeV}$ centre-of-mass energy with the ATLAS detector, Eur. Phys. J. C 71 (2011) 1512 [arXiv:1009.5908] [SPIRES].

[2] CMS collaboration, V. Khachatryan et al., Search for Dijet Resonances in $7 \mathrm{TeV} p p$ Collisions at CMS, Phys. Rev. Lett. 105 (2010) 211801 [arXiv:1010.0203] [SPIRES].

[3] U. Baur, I. Hinchliffe and D. Zeppenfeld, Excited Quark Production at Hadron Colliders, Int. J. Mod. Phys. A 2 (1987) 1285 [SPIRES]. 
[4] S. Cullen, M. Perelstein and M.E. Peskin, TeV strings and collider probes of large extra dimensions, Phys. Rev. D 62 (2000) 055012 [hep-ph/0001166] [SPIRES].

[5] L.A. Anchordoqui et al., Dijet signals for low mass strings at the LHC, Phys. Rev. Lett. 101 (2008) 241803 [arXiv:0808.0497] [SPIRES].

[6] S. Frixione and B.R. Webber, Matching NLO QCD computations and parton shower simulations, JHEP 06 (2002) 029 [hep-ph/0204244] [SPIRES].

[7] P. Nason, A new method for combining NLO QCD with shower Monte Carlo algorithms, JHEP 11 (2004) 040 [hep-ph/0409146] [SPIRES].

[8] S. Frixione, P. Nason and C. Oleari, Matching NLO QCD computations with Parton Shower simulations: the POWHEG method, JHEP 11 (2007) 070 [arXiv: 0709. 2092] [SPIRES].

[9] S. Frixione, F. Stoeckli, P. Torrielli, B.R. Webber and C.D. White, The MCaNLO 4.0 Event Generator, arXiv:1010.0819 [SPIRES].

[10] M. Bahr et al., HERWIG++ Physics and Manual, Eur. Phys. J. C 58 (2008) 639 [arXiv:0803.0883] [SPIRES].

[11] S. Hoche, F. Krauss, M. Schonherr and F. Siegert, Automating the POWHEG method in Sherpa, arXiv: 1008.5399 [SPIRES].

[12] The processes implemented so far in the POWHEG BOX are available at the svn repository: svn://powhegbox.mib.infn.it/trunk/POWHEG-BOX.

[13] S. Alioli, P. Nason, C. Oleari and E. Re, A general framework for implementing NLO calculations in shower Monte Carlo programs: the POWHEG BOX, JHEP 06 (2010) 043 [arXiv: 1002 .2581] [SPIRES].

[14] S. Frixione and G. Ridolfi, Jet photoproduction at HERA, Nucl. Phys. B 507 (1997) 315 [hep-ph/9707345] [SPIRES].

[15] S. Frixione, Z. Kunszt and A. Signer, Three jet cross-sections to next-to-leading order, Nucl. Phys. B 467 (1996) 399 [hep-ph/9512328] [SPIRES].

[16] S. Frixione, A General approach to jet cross-sections in QCD, Nucl. Phys. B 507 (1997) 295 [hep-ph/9706545] [SPIRES].

[17] R.K. Ellis and J.C. Sexton, QCD radiative corrections to parton-parton scattering, Nucl. Phys. B 269 (1986) 445 [SPIRES].

[18] Z. Kunszt and D.E. Soper, Calculation of jet cross-sections in hadron collisions at order $\alpha_{s}^{3}$, Phys. Rev. D 46 (1992) 192 [SPIRES].

[19] R.K. Ellis, G. Marchesini and B.R. Webber, Soft Radiation in Parton Parton Scattering, Nucl. Phys. B 286 (1987) 643 [SPIRES].

[20] G. 't Hooft, A planar diagram theory for strong interactions, Nucl. Phys. B 72 (1974) 461 [SPIRES].

[21] G. Marchesini and B.R. Webber, Monte Carlo Simulation of General Hard Processes with Coherent QCD Radiation, Nucl. Phys. B 310 (1988) 461 [SPIRES].

[22] S. Alioli, P. Nason, C. Oleari and E. Re, Vector boson plus one jet production in POWHEG, JHEP 01 (2011) 095 [arXiv: 1009. 5594] [SPIRES].

[23] G.C. Blazey et al., Run II jet physics, hep-ex/0005012 [SPIRES]. 
[24] J. Pumplin et al., New generation of parton distributions with uncertainties from global QCD analysis, JHEP 07 (2002) 012 [hep-ph/0201195] [SPIRES].

[25] M. Cacciari and G.P. Salam, Dispelling the $N^{3}$ myth for the $k_{T}$ jet-finder, Phys. Lett. B 641 (2006) 57 [hep-ph/0512210] [SPIRES].

[26] M. Cacciari, G.P. Salam and G. Soyez, http://www.fastjet.fr

[27] G.P. Salam and G. Soyez, A practical Seedless Infrared-Safe Cone jet algorithm, JHEP 05 (2007) 086 [arXiv:0704.0292] [SPIRES].

[28] S.D. Ellis and D.E. Soper, Successive combination jet algorithm for hadron collisions, Phys. Rev. D 48 (1993) 3160 [hep-ph/9305266] [SPIRES].

[29] S. Catani, Y.L. Dokshitzer, M.H. Seymour and B.R. Webber, Longitudinally invariant $k_{T}$ clustering algorithms for hadron-hadron collisions, Nucl. Phys. B 406 (1993) 187 [SPIRES].

[30] P. Nason, MINT: a Computer Program for Adaptive Monte Carlo Integration and Generation of Unweighted Distributions, arXiv:0709.2085 [SPIRES].

[31] CDF collaboration, T. Aaltonen et al., Measurement of the Inclusive Jet Cross section at the Fermilab Tevatron p $\bar{p}$ Collider Using a Cone-Based Jet Algorithm, Phys. Rev. D 78 (2008) 052006 [arXiv:0807.2204] [SPIRES].

[32] M. Klasen and G. Kramer, Dijet cross-sections at $O\left(\alpha \alpha_{S}^{2}\right)$ in photon-proton collisions, Phys. Lett. B 366 (1996) 385 [hep-ph/9508337] [SPIRES].

[33] A. Banfi and M. Dasgupta, Dijet rates with symmetric $E_{T}$ cuts, JHEP 01 (2004) 027 [hep-ph/0312108] [SPIRES].

[34] D0 collaboration, V.M. Abazov et al., Measurement of the dijet invariant mass cross section in proton anti-proton collisions at $\sqrt{s}=1.96 \mathrm{TeV}$, Phys. Lett. B 693 (2010) 531 [arXiv: 1002 .4594] [SPIRES].

[35] M. Rubin, G.P. Salam and S. Sapeta, Giant QCD K-factors beyond NLO, JHEP 09 (2010) 084 [arXiv: 1006.2144] [SPIRES].

[36] S. Alioli, P. Nason, C. Oleari and E. Re, NLO Higgs boson production via gluon fusion matched with shower in POWHEG, JHEP 04 (2009) 002 [arXiv:0812.0578] [SPIRES].

[37] K. Hamilton, P. Richardson and J. Tully, A Positive-Weight Next-to-Leading Order Monte Carlo Simulation for Higgs Boson Production, JHEP 04 (2009) 116 [arXiv:0903.4345] [SPIRES].

[38] T. Sjöstrand, S. Mrenna and P.Z. Skands, PYTHIA 6.4 Physics and Manual, JHEP 05 (2006) 026 [hep-ph/0603175] [SPIRES].

[39] G. Corcella et al., HERWIG 6.5: an event generator for Hadron Emission Reactions With Interfering Gluons (including supersymmetric processes), JHEP 01 (2001) 010 [hep-ph/0011363] [SPIRES].

[40] G. Corcella et al., HERWIG 6.5 release note, hep-ph/0210213 [SPIRES].

[41] E. Boos et al., Generic user process interface for event generators, hep-ph/0109068 [SPIRES].

[42] J. Alwall et al., A standard format for Les Houches event files, Comput. Phys. Commun. 176 (2007) 300 [hep-ph/0609017] [SPIRES]. 
[43] K. Hamilton, P. Richardson and J. Tully, A Positive-Weight Next-to-Leading Order Monte Carlo Simulation of Drell-Yan Vector Boson Production, JHEP 10 (2008) 015 [arXiv:0806.0290] [SPIRES].

[44] T. Sjöstrand and P.Z. Skands, Multiple interactions and the structure of beam remnants, JHEP 03 (2004) 053 [hep-ph/0402078] [SPIRES].

[45] CDF - Run II collaboration, A. Abulencia et al., Measurement of the Inclusive Jet Cross

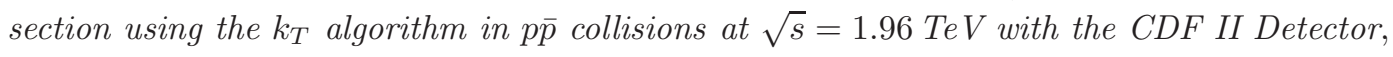
Phys. Rev. D 75 (2007) 092006 [hep-ex/0701051] [SPIRES].

[46] D0 collaboration, V.M. Abazov et al., Measurement of dijet azimuthal decorrelations at central rapidities in pp collisions at $\sqrt{s}=1.96$ TeV, Phys. Rev. Lett. 94 (2005) 221801 [hep-ex/0409040] [SPIRES].

[47] CDF collaboration, F. Abe et al., Evidence for color coherence in p $\bar{p}$ collisions at $\sqrt{s}=1.8$ TeV, Phys. Rev. D 50 (1994) 5562 [SPIRES].

[48] M. Cacciari, G.P. Salam and G. Soyez, The anti- $k_{T}$ jet clustering algorithm, JHEP 04 (2008) 063 [arXiv:0802.1189] [SPIRES]. 